\title{
Gradhiva
}

\section{Double enquête. Une leçon d'écriture chamanique en Amazonie}

Double Inquiry. A Lesson in Shamanic Writing in the Amazon

\section{Andrea-Luz Gutierrez-Choquevilca}

\section{(2) OpenEdition}

1 Journals

Édition électronique

URL : https://journals.openedition.org/gradhiva/5416

DOI : 10.4000/gradhiva.5416

ISSN : 1760-849X

Éditeur

Musée du quai Branly Jacques Chirac

Édition imprimée

Date de publication : 24 mars 2021

Pagination : 60-81

ISBN : 978-2-35744-132-3

ISSN : 0764-8928

\section{Référence électronique}

Andrea-Luz Gutierrez-Choquevilca, « Double enquête. Une leçon d'écriture chamanique en Amazonie », Gradhiva [En ligne], 32 | 2021, mis en ligne le 02 avril 2021, consulté le 25 mai 2021. URL : http://

journals.openedition.org/gradhiva/5416; DOI : https://doi.org/10.4000/gradhiva.5416

(c) musée du quai Branly 


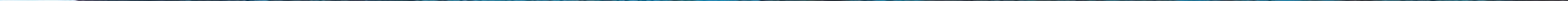


Double enquête Une leçon d'écriture chamanique

en Amazonie

$$
\begin{gathered}
\text { Andrea-Luz } \\
\text { Gutierrez-Choquevilca }
\end{gathered}
$$


Lors d'un rêve initiatique, un chamane amérindien affirme avoir reçu de «Dieu» en personne l'injonction de coucher sur papier les voix mystérieuses entendues au cours de ses visions: les esprits supay lui apparaissent sous la forme de «créatures lettres» étincelantes, identifiées comme la trace d'un monde invisible révélé. À son réveil, EW écri† ses chants de rêve ikara inspirés par les esprits. Sa biblioteca clandestine compte des dizaines de manuscrits et de feuilles déchirées, froissées, raturées. Qu'advient-il lorsque des sociétés dont la mémoire rituelle semblait, jusque-là, réservée à l'oralité, ont recours à la possibilité d'inscrire des voix révélées sous la forme d'une trace figée, détachée de son contexte d'énonciation originel? Ce texte explore l'avènement et l'épistémologie de cette cryptographie en haute Amazonie. Le récit de la rencontre entre le chamane et l'anthropologue dévoile le geste complexe qui préside à l'inscription et au déchiffrement de ces textes poétiques. S'il est prêté aux chants ikara le pouvoir de sceller des pactes, de guérir ou de transformer l'ordre du monde, l'écrit est devenu pour EW la trace d'un corps, celui des petites âmes aya runa et des esprits supay. Une conception radicale de la traduction, opérant tant sur des signes que sur des êtres, met au jour une ontologie amérindienne du langage fondée sur la transformation.

\section{VERTIGES DE L'ENQUÊTE}

20 juillet 2011. Iquitos, quartier Santa Clara, Amazonie péruvienne. Il fait nuit. Le sol est jonché de papiers volants éparpillés sur le tapis où viennent d'habitude, l'un après l'autre, s'étendre les patients, pour recevoir les incantations ikara soufflées par le maître. EW habite ici. La petite maison aux murs de briques inachevés se dresse dans un quartier clairsemé de la périphérie d'Iquitos. Autour, le bourdon des musiques de radios tropicales diffuse au loin, sous le halo grouillant des réverbères, des notes qui grésillent. Les yeux sombres d'E.W brillent sous la lumière blafarde du néon. Il est assis sur un tabouret en bois un peu bancal dans un coin de la pièce, un carnet sur les genoux, un stylo d'écolier vert dans la main.

- Kayna tutapish shamushkanki pani manachu

«Tu es venue hier, n'est-ce pas?»

Je reste immobile, dans l'attente d'une explication.

- Ari kayna tutapish tusushpaykika taaakihushkanki talalalan den den den den den day day day day

«Oui... la nuit dernière... tu dansais et tu as commencé à chanter talalalan den den den den den day day day day... »
Silence. Un malentendu, sans doute. Je n'étais pas ici, j'étais ailleurs. Je dormais.

EW prend un air sérieux et me tend une feuille manuscrite sur laquelle j'aperçois quelques mots griffouillés en lettres capitales à l'encre noire. Il est un peu aǵité. Une cérémonie d'ayahuasca a eu lieu la veille, réunissant plusieurs chamanes runa et cocama, qui échangent des recettes de remèdes, des chants de visions, des sortilèges et des envoûtements. Lors d'une vision, EW m'a trouvée là sous une apparence éthérée mais vivante qu'il nomme muskuy, le «rêve». Dans le souvenir de ce rêve qu'il me décrit, je participais à la cérémonie, j'étais assise à côté de lui puis je me levais pour danser autour des participants:

- Kiiikin shutillami muskuchirwashkanki pani. Chaypimi aysawashkanki, suuuni ruku chukchayu kanka rikurishpa muru pampalliyu, shushuhushpaykika, chasna shushuuu... urkumanta aya runa shimipi takihushpa, Inga shimipi rimashpayki nishpa chari.

«Tu m'as fait faire un rêve [muskuchirea-], un rêve limpide et clair, un rêve véritable, ma sœur. Là, tu m'as pris par la main, tu avais de longs cheveux et une jupe chamarrée, tu sifflais comme cela: sssh $s s s h$, tu parlais la langue des vieux morts de la cordillère, la langue des Incas, sans doute». 


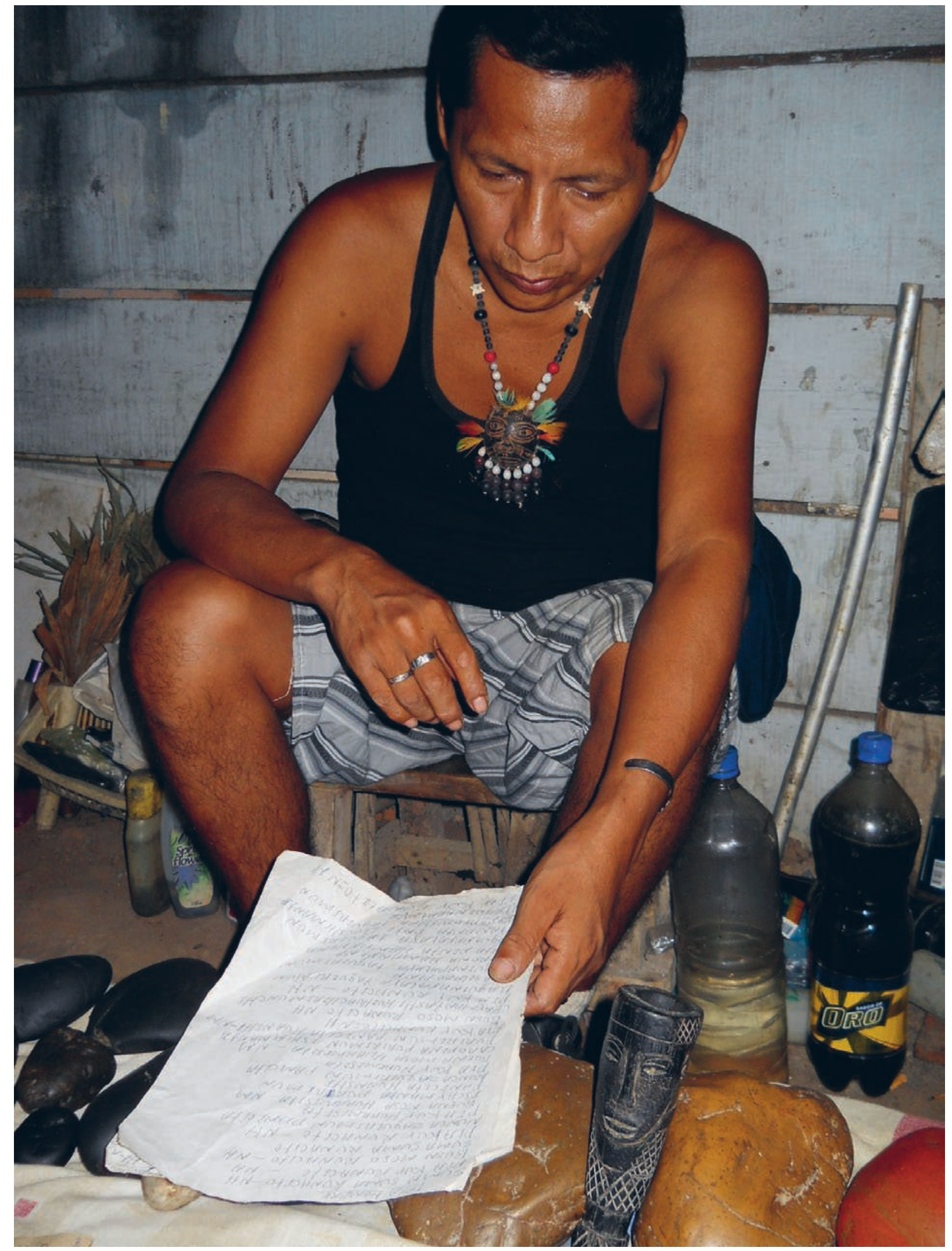

EW dans la pièce où il officie, montre une paperole sur laquelle il souffle un chant $i k a r a$, entouré de ses pierres magiques. Photo: Andrea-Luz Gutierrez-Choquevilca, 2011 
-Mmh? Imatash?

«Ah bon?»

- Mana... Shuti pani! Shutilla rikurinki muskuynipi imanash... suma warmisita chil chil chil tushunki talaaaan machaynipi pukuwashkanki sumakta chay takinata aya runamanta uyarirayahun

«Non... c'est vrai, ma sœur! Ton image est limpide dans mon rêve, comme cela: une jolie petite femme chil chil chil [cliquetis des sonnailles, apparition], tu danses, talaaaaan [son du corps d'EW qui s'étend au sol, signalant le début de l'état de narcose], dans mon ivresse tu m'as bien soufflé [pukuwa-] ce chant des images spectrales des vieux morts, le chant de ta voix résonne encore.»

Il rêve que je lui apparais. Il rêve que je danse, que je chante, pour lui, que je lui enseigne un chant en soufflant sur sa tête, pour qu'il devienne plus puissant. Au petit matin, repu de son ivresse et courbaturé, il écrit le chant qu'il a entendu sur une feuille volante. Quelques heures plus tard, il me donne la feuille en question (ma traduction à droite) :

$\begin{array}{ll}\begin{array}{l}\text { SERRANITA SERRANITA } \\ \text { SERRANITA SERRANITA } \\ \text { SERRANITA WARMISTA }\end{array} & \begin{array}{l}\text { Petite femme des hautes terres } \\ \text { Petite femme des hautes terres } \\ \text { Petite femme des hautes terres } \\ \text { petite femme }\end{array} \\ \begin{array}{l}\text { KUSKENITA KUSKENITA } \\ \text { KUSKENITA KUSKENITA }\end{array} & \begin{array}{l}\text { Petite femme de Cuseo } \\ \text { KUSKENITA WARMISTA }\end{array} \\ \begin{array}{l}\text { Petite femme de Cusco petite } \\ \text { femme }\end{array} \\ \text { PAMPENITA PAMPENITA } & \text { Petite jupe } \\ \text { PAMPENITA PAMPENITA } & \text { Petite jupe } \\ \text { PAMPENITA WARMISTA } & \text { Petite femme à la petite jupe }\end{array}$

SANGAPILLA SANGAPILLA Plante de charme sankapilla SANGAPILLA SANGAPILLA Plante de charme sankapilla SANGAPILLA WARMISTA Petite femme de la plante SANGAPILLA WARMISTA de charme sankapilla bis

$\begin{array}{ll}\text { VAYNILLA VAYNILLA } & \text { Vanille } \\ \text { VAYNILLA VAYNILLA } & \text { Vanille } \\ \text { VAYNILLA WARMISTA } & \text { Petite femme de la vanille } \\ & \\ \text { BONBINSANA BONBINSANA } & \text { Arbre bobinsana } \\ \text { BONBINSANA BONBINSANA } & \text { (Calliandra angustifolia) } \\ \text { BONBINSANA FRAGUIANSITA } & \text { Arbre bobinsana }\end{array}$
(Calliandra angustifolia) Petit parfum de l'arbre bobinsana

$\begin{array}{ll}\text { KUSKENITA KUSKENITA } & \text { Petite femme de Cusco } \\ \text { KUSKENITA KUSKENITA } & \text { Petite femme de Cusco } \\ \text { KUSKENITA FRAGUIANSITA } & \text { Petit parfum de la petite femme } \\ & \text { de la cordillère }\end{array}$

SERRANITA SERRANITA SERRANITA SERRANITA SERRANITA FRAGUIANSITA
Le chant, écrit en lettres capitales à la façon de caractères imprimés, est une citation de ma propre voix entendue par EW en rêve, et que j'ignorais, avant qu'elle ne soit pour la première fois énoncée par mon ami, ventriloque. EW me confie qu'il s'agit d'une incantation destinée à sceller des pactes d'amour et d'attraction pusanka ou watana et à déjouer les sortilèges puissants des chamanes ennemis. La «petite jupe» que je porte est une arme onirique qui se déploie à la manière d'un éventail. Sa couleur est semblable, me dit-il, au plumage de l'oiseau caurale-soleil tankrilla (Euripiga helias). Déployé, celui-ci laisse apparaître sur chaque aile une tache rouge cerclée de noir comme un œil géant. «llipia llipia» est le son identifiant cette apparition dans le rêve. Les parfums végétaux sankapilla, bobinsana, l'odeur de vanille (rare en Amazonie) doivent agir secrètement sur les intentions des destinataires de ce chant. Présent mais invisible, le parfum opère à la façon d'un toucher sans contact, suave mishkiiiilla, au point qu'il aurait le pouvoir de distraire l'ennemi. Du point de vue de sa composition, le chant est une variante classique du répertoire des chants chamaniques ikara qu'EW maîtrise. Il débute par l'invocation d'une entité, dont EW décline successivement les attributs: jupe, parfum, formes végétales, lieu d'origine. Il la nomme et l'invoque par une série de métonymies évoquant diverses facettes sensorielles, morphologiques ou topologiques. Une logique du concret s'applique ici au langage rituel (Gutierrez-Choquevilca 2017). Ce chant est par ailleurs une citation, celle de la voix de son énonciateur onirique. En quechua, le terme le plus couramment employé est kaya-chi( «faire appeler») ou encore kati-chi- («répéter, faire suivre, faire répondre») en assumant la voix d'un d'autre, dans un dispositif énonciatif polyphone.

D'un geste bref de la main, EW secoue légèrement la feuille portant les inscriptions et la pose délicatement sur le tapis où gisent éparpillées des dizaines d'autres feuilles jaunies, cornées, déchirées, écrites par lui ou d'autres initiés. Des centaines de textes, dont il a soiǵneusement omis de me siǵnaler l'existence depuis mon arrivée, sont ici rassemblés dans un désordre orǵanisé comme une danse. Les feuillets s'agitent et se délitent pour les plus anciens, lorsqu'il fouille d'une main furtive, d'autres se plient, se déplient, à moitié déroulés. Je distingue de l'encre rouge, bleue, des ratures, des noms gribouillés comme des insectes piqués sur le papier, de simples pages de carnets d'écoliers arrachées, des blocs de papier en fibre de banane couverts d'écritures au stylo bille rouǵe. Voici la biblioteca d'EW, sa «bibliothèque» de chants de rêve ikara. Avec ça, EW soigne, il combat les sortilèges ennemis, il tue et scelle des pactes d'amour. Le chamanisme est un savoir qui s'apprend et qui s'écrit. Ces papiers recouverts de paroles énoncées dans un rêve, puis inscrites sur un support qu'il nomme killkarishkata («les choses écrites»), sont la trace de ce rêve polyphone.

La confidence faite par EW m'avait paru tout d'abord insignifiante. Elle soulève cependant deux éniǵmes. La première concerne la place qu'occupe l'anthropologue dans les savoirs qu'il prétend de loin observer. Elle interroge les conditions d'objectivation 


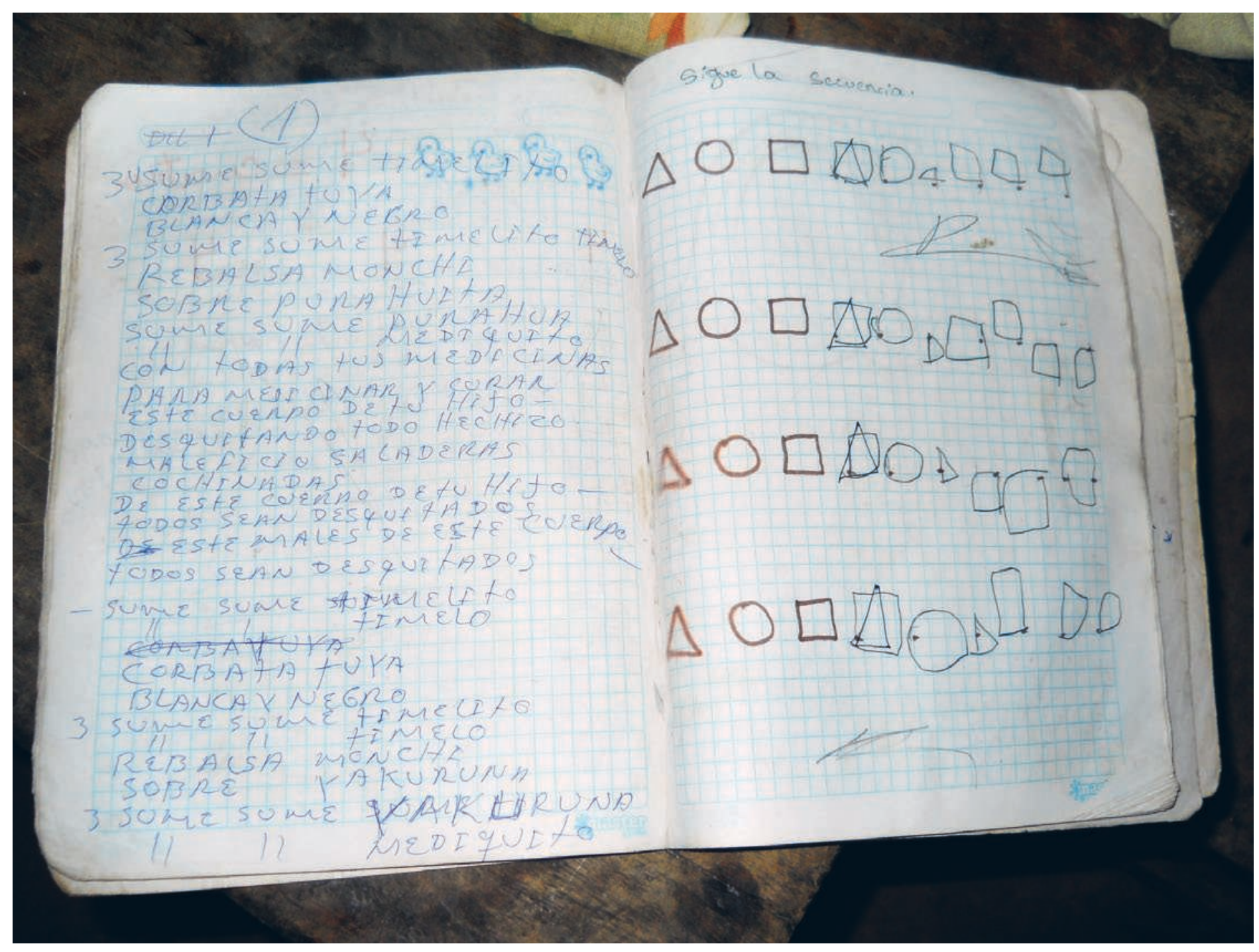

Chant du vanneau de Cayenne «timelito» à la cravate blanche et noire en espagnol, sur l'un des carnets killkarishkata. Photo: Andrea-Luz Gutierrez-Choquevilca, 2011.

du savoir dont l'enquête propose de restaurer les indices, le squelette. Qu'arrive-t-il quand l'observateur est observé au point de devenir, dans le monde nocturne de ses sujets d'observation, une source d'inspiration poétique? Les témoignnages signalant la relation intime entre l'ethnologue et son double sont légion. Couché dans les carnets oubliés d'un ethnologue, le rêve d'une jeune femme aché, Baipurangii, faisant l'amour à Lucien Sebag (1964, rêve n 121), m'a laissé une impression vive d'incursion postmoderne avant l'heure. Les désirs d'enfant inavoués de Baipuranǵi et les pensées mélancoliques de Sebaǵ trouvent dans le lanǵaǵe du rêve une expression possible en deçà de toute enquête et au-delà des barricades posées par l'objectivation scientifique. Dans le récit de rêve, les voix de Baipuranǵi et de Sebag se côtoient dans un dialogue utopique. Le rêve donne chair au lien qui les unit, il infléchit leur enquête respective et lui confère une tonalité tragique: «Ce serait alors notre mort commune que le rêve décrirait [...]. C'est sur sa mort et la mienne que Baipurangi se lamenterait. Mais il n'est pas possible d'arriver ici à une quelconque certitude», écrivait-il (Leavitt 2005: 109). Le rêve offre un métalangage permettant à chacun de représenter et d'agir sur les conditions de l'enquête. Plusieurs décennies plus tard, une femme chamane zapara nommée Kiawka rêve de l'ethnologue Anne-Gaël Bilhaut qui tourbillonne sur un hélicoptère brillant, puis dans un avion (Bilhaut 2011: 158). Kiawka converse avec l'esprit de l'anaconda, puis elle se réveille et téléphone à l'ethnologue pour lui conter son rêve et les avancées de son enquête sur la «mémoire» zapara. Un autre rêve me surprend. Kiawka, devenue visionnaire-scientifique, brandit son «boa-Dictaphone» pour capturer la voix des ancêtres : la voix entre par la bouche du boa, lequel enregistre par la queue (Bilhaut 2011: 236).

La réflexivité de l'enquête prend des proportions vertigineuses. Que l'ethnographe et son alter ego soient autant affectés l'un que l'autre par la rencontre humaine qui se joue dans l'enquête, voilà une évidence (FavretSaada 1990). Le métalangage qui s'élabore en filigrane doit être cependant examiné. Avec la confidence d'EW, une leçon d'écriture chamanique m'est offerte à laquelle j'ai pris part sans le vouloir, devenant à mon tour sujet et objet du savoir. EW voit en moi une étrangère et une alliée qui parle sa langue. Je lui apparais en esprit auxiliaire spectral féminin aya runa warmi sifflant et chantant la langue des morts. EW mène son enquête sur ma personne, depuis mon arrivée. Il sait que je sais quelque chose. Je sais qu'il sait autre chose. Il est intrigué par ma réticence à m'engager dans un rapport d'initiation et de consommation narcotique,
1. «Elle fait l'amour avec moi. Je lui parle: nde neby kylaijue Et dans son rêve elle dit qu'elle désirait des enfants Nous faisons l'amour Etensuite we watakuari Elle conte toute l'histoire à sa mère Baipugi qu'elle rencontre Wuchugi sho meno ve», Fonds Lucien Sebag, Laboratoire d'anthropologie sociale (ma transcription d'une page de carnet de terrain de Sebag avec l'annotation des rêves de Baipurangi, figure 4, Leavitt 2005). 


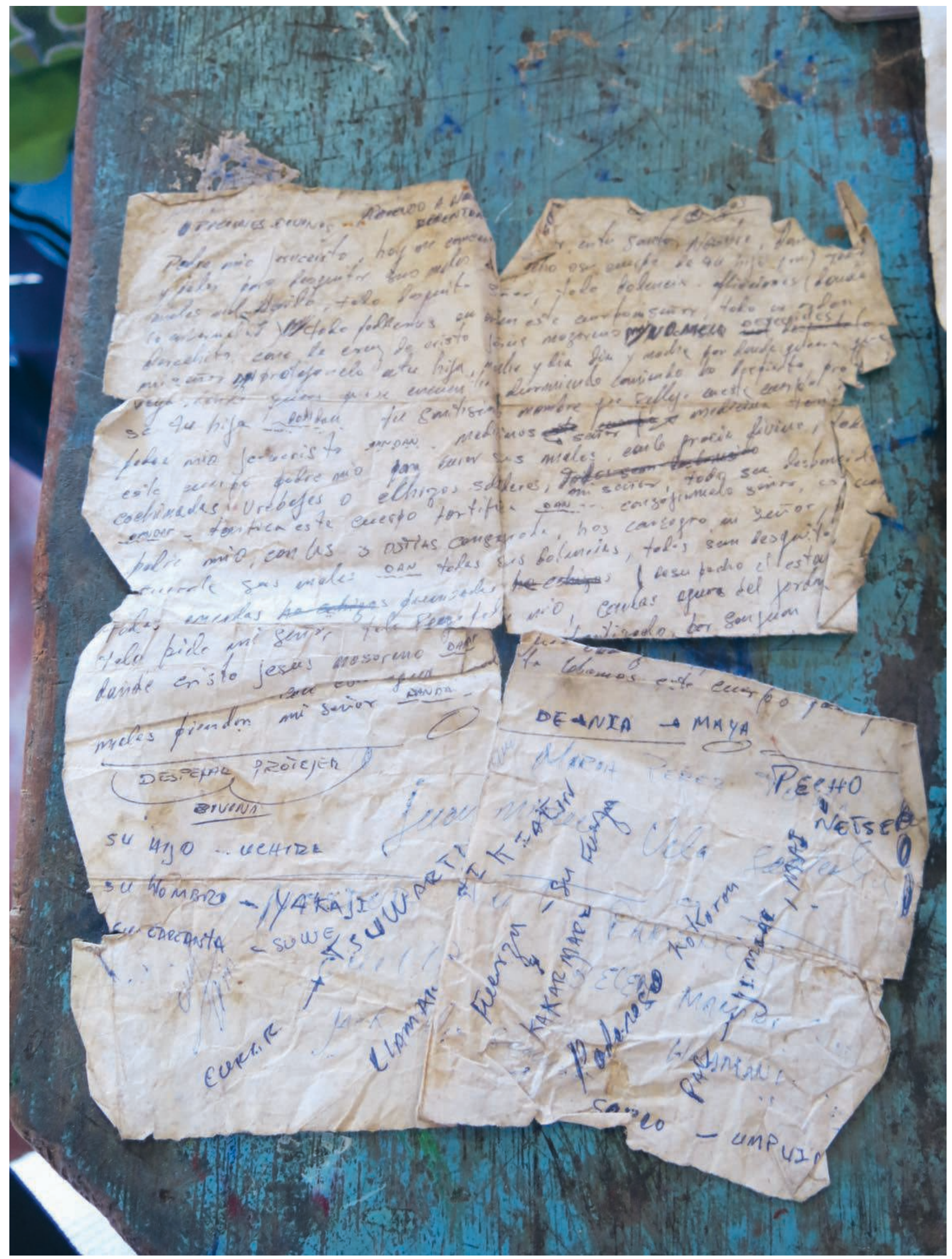

Paperole contenant un sortilège en espagnol, traduction d'un lexique achuar et des noms de patients ou victimes effacés. Photo: Andrea-Luz Gutierrez-Choquevilca, 2011. 
prisé par les touristes étrangers. Je participe depuis plusieurs semaines aux séances de cure avec les patients du quartier Santa Clara, aux cérémonies avec d'autres chamanes de la région, je note dans mes carnets et j'enregistre sans répit. Quelle coïncidence y a-t-il entre son rêve et mon enquête? L'autre jour, EW m'a interpelée en m'accusant de lui cacher des choses. Des chants que j'aurais entendus sur le Pastaza, un autre fleuve où j'ai mené mon enquête. Je lui propose de lui faire entendre mes enregistrements, mais il souhaite que je chante pour lui. Je réponds de façon évasive. Nous doutons autant l'un que l'autre de l'issue de nos échanges: j'avance à tâtons dans la compréhension de son savoir. Il observe. C'est peut-être dans cette économie singulière du dit et du non-dit, de l'observation réciproque que prend forme le savoir anthropologique. On trouve là différentes strates de savoirs sédimentés au cours de nos enquêtes converǵentes. Un malentendu productif s'est installé entre nous, qui n'est ni celui qui lie le patient au chamane, ni celui qui informe la relation entre le disciple et le maître. $\mathrm{EW}$ attend quelque chose, tout comme j'espère bientôt détenir une clef pour décrypter le caractère inexplicable de ses chants. Nous sommes tous deux confondus dans l'attente d'un dévoilement. Ce qui varie, entre nous, c'est la modalité du déchiffrement. Voir, siffler, chanter, entendre, communiquer en rêve ou transmettre par la parole.

\section{GONQUÊTE DE L'ÉGRITURE}

La découverte du chant de rêve confié par EW pose une seconde éniǵme, celle de la transformation contemporaine des modes de connaissance amérindiens. La conquête de l'écriture par des sociétés longtemps considérées comme les derniers «peuples sans écriture» intriǵue les anthropoloǵues ${ }^{2}$. La célèbre «leçon d'écriture » d'un indien nambikwara traçant des lignes ondulées sur le carnet de Claude Lévi-Strauss continue d'éveiller la spéculation. S'aǵissait-il d'une vulgaire imitation, d'une quête d'autorité et de prestige que le chef Nambikwara aurait immédiatement perçus dans la fonction de l'écriture occidentale? L'abandon du chef par sa troupe avait-il pour origine l'intuition que l'écriture des blancs portait en elle-même le germe de l'exploitation de l'homme par l'homme? Une bibliothèque improbable vient à l'esprit, rassemblant les rares écrits accessibles aux peuples de la forêt tropicale, au cours de plusieurs siècles de rencontres et d'exploitation. Ce sont des bibles, des livres de prière, des fascicules de magie imprimés en Espagne, égarés dans les malles de soldats et de commerçants, des documents d'état civil ou des certificats de baptême, des feuilles d'inventaires d'objets cédés par les patrons aux Indiens en échange du travail dans l'extraction de l'hévéa ou dans la cocaïne, des carnets d'écolier aux pages déchirées, des papiers d'ethnologues oubliés... L'écriture est partout. Cependant, à l'exception de quelques anecdotes et d'un essai d'anthropologie inversée signé Pierre Déléage (2017), peu d'attention a été consacrée à l'impression vive que l'écriture laisse dans les esprits des peuples des basses terres ${ }^{3}$.
Quelle forme revêt la diffusion de l'écriture alphabétique? En Amazonie comme dans les Andes, une pratique de la literacy se déploie et s'autonomise à la faveur de la traduction en alphabet latin de certaines langues amérindiennes par les missionnaires jésuites, dominicains, puis par une vague de missionnaires évanǵélistes de l'Instituto Lingúuístico de Verano (ILV) ${ }^{4}$ (Steward 1948: 510; Gow 2009; Neuman 2012; Platt 1992: 137; Stoll 2005). Cette diffusion sélective de l'écriture concerne principalement des textes chrétiens ou administratifs et des lettres officielles; elle se fonde sur un vaste système de relations politiques et diplomatiques établies avec les évangélisateurs et l'administration coloniale des pays sud-américains. À la fin du XIX ${ }^{\mathrm{e}}$ siècle, pendant la période du boom du caoutchouc (1880-1915), le système vient inclure les relations que les Amérindiens établissent avec les barons du caoutchouc, les patrones. Ce n'est qu'à partir de la seconde moitié du $\mathrm{xx}^{\mathrm{e}}$ siècle, avec le renfort des institutions scolaires, que l'écriture alphabétique infiltre le quotidien des sociétés amérindiennes, à des rythmes variables selon les régiions. Les documents officiels deviennent alors un instrument de communication diplomatique avec les exploitants des ressources tropicales et les représentants locaux des États sud-américains à mesure que s'institutionnalisent les formes d'autorité politique en Amazonie (Brown 1993 : 312; Taussig 1987; Hugh Jones 1992 : 48-53 et 2010 : 207-208). On voit circuler dès les années 1970 abécédaires et manuels, journaux, bibles, certificats, laissez-passer sur les territoires des communautés, feuillets extraits de libros de acta des fédérations amérindiennes, titres de propriété, requêtes écrites adressées aux institutions étatiques ou privées.

Toutefois, la maîtrise de l'écriture alphabétique et de la lecture reste inégale et souvent l'apanage d'un petit nombre investi d'une fonction de scribe ou secretario dans le cadre des organisations fédératives. L'écriture n'est pas tant mobilisée comme une mnémotechnique visant à se substituer à la mémoire orale que pour ses effets performatifs, en tant qu' «acte». La maîtrise de l'usage bureaucratique de l'écrit reste intimement liée à l'accès à des ressources matérielles - marchandises ou territoires - et idéologiques ${ }^{5}$.

Cependant, un usaǵe maǵique de l'écriture est attesté en Amazonie dès la fin du XIx ${ }^{\mathrm{e}}$ siècle, parallèlement à celui des documents officiels. Les rares documents reliés - livres, journaux, feuillets - que se procurent les Amérindiens se voient alors intégrés à un savoir spécifique, les visions, au point de figurer comme la pierre de voûte de rituels d'initiation chamanique et, dans quelques cas plus rares, de mouvements prophétiques (Déléage 2010 et 2017: 122; Gow 2001: 191-218). Vers la fin des années 1870 en Guyane britannique, on voit apparaître dans les visions d'un prophète amérindien une bible, des livres ou papiers «venus du ciel», chez les Akawaïo (Kapon) et Macuxi (Pemon), à l'origine de l'institutionnalisation du mouvement prophétique Alleluia (Déléage 2017 : 124; Azevedo 2004 : 107 ; Butt Colson 1971: 32-48 ). Dans le Nord-Ouest amazonien, la fascination des Ticuna pour la Bible et l'outil livresque contribue au prestige de nouveaux leaders religieux. La remise de la version
2. Claude Lévi-Strauss avait jugé bon, en 1954, que l'on renommât sa chaire consacrée aux peuples amérindiens des basses terres à la Vesection des basses terres à la $V$ e section
de l'École pratique des hautes études «Religions des peuples sans écriture».

3. Parmi les exceptions notables, voir: Déléage 2010 et 2017; Gow 1990; Wogan 1994; 2017; Gow 1990; Wogan 1994;
Hugh-Jones 2010; Bacigalupo Hugh-Jones 2010; Bacigalupo
2014; Allard et Walker 2016; et en Amérique du nord: Axtell 1985.

4. L'écriture joue un rôle notoire dans la conquête des âmes. La traduction des textes chrétiens dans les langues amérindiennes est indispensable à la colonisation de l'imaginaire religieux. L'instruction religieux. Linstruction
de traducteurs amérindiens se heurte cependant à la diversité linguistique et à l'incommunicabilité avérée entre des peuples dispersés dans l'espace. La langue quechua se diffuse progressivement comme une lingua franca en Amazonie péruvienne et équatorienne et fait place, dans les vallées du fleuve Pastaza, Napo et Curaray de la mission de Maynas, à l'ethnogénèse de sociétés hybrides qualifiées d'indios cristianos, bénéficiant d'une protection contre les incursions esclavagistes (Gutierrez-Choquevilca 2011).

5. Avec la prolifération de documents administratifs, l'écriture prend place dans une série d'échanges caractérisés par une temporalité spécifique (une réciprocité «différée») et un formalisme accru. Elle s'inscrit dans un continuum de relations asymétriques engagées avec des interlocuteurs dominants «blancs» ou métis: missionnaires, trafiquants de cocaïne, barons du caoutchouc, représentants de l'État (Brown 1993: 312; Hugh Jones 1992: 48; Gúzman Gallegos 2009; Gordillo 2006; Allard et Walker 2016; Nahum-Claudel 2016). 


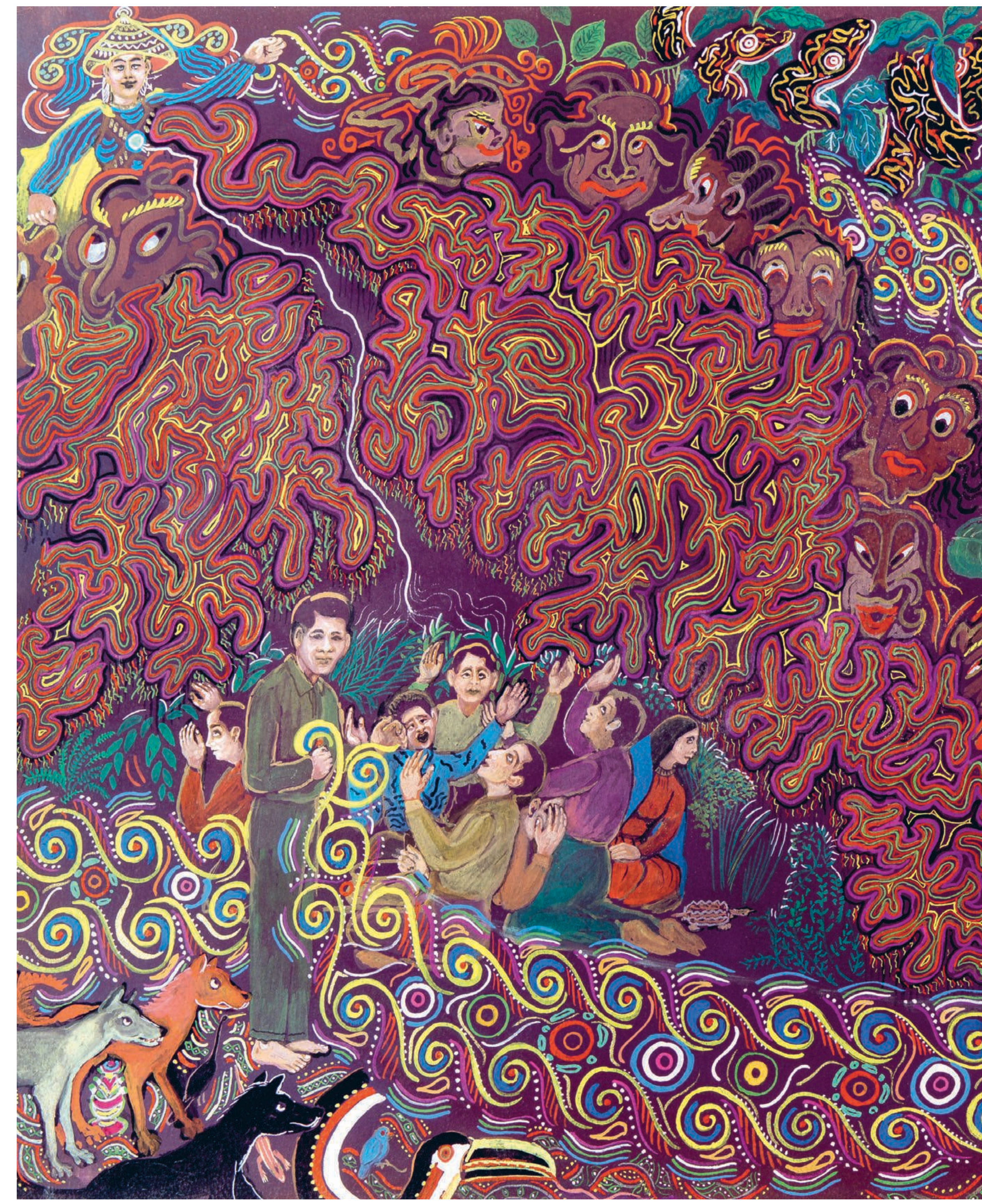


6. Sur le rôle du livre dans les mouvements messianiques tikuna, voir: Goulard 2000: 287-291 et 290, n.32.

7. Le lecteur consultera les travaux de Pierre Déléage dont l'immense mérite est de systématiser les données sur la question $(2010 ; 2017)$

8. Brugmansia suaveolens, Solanaceae. définitive de la Bible traduite en langue ticuna par un pasteur évangéliste donne lieu à une cérémonie générant une attente rituelle chez les convertis, eux-mêmes illettrés ${ }^{6}$. Un fétichisme rituel accompaǵne la réception de l'écrit biblique. Il converge avec les préceptes théologiques fondamentalistes de l'Institut linguistique d'été, accordant à la traduction du message divin une aǵentivité singulière: "L'ILV ne se considère pas lui-même comme une mission, parce que ce sont les traductions de la Bible elle-même, et non ses membres, qui sont la cause des conversions spirituelles. » (Stoll 1985: 24) La figure du linguiste traducteur se substitue à celle de l'évangélisateur et fixe les conditions de la lecture du texte divin et de son inscription. Autre coïncidence. Les normes scripturaires imposées par les missionnaires saturent le texte biblique de néologismes et de formes syntaxiques inusitées, au point d'introduire une opacité dans l'appréhension que les Amérindiens ont de ce texte. Le livre biblique offre des distorsions linguistiques présentant donc quelques similitudes avec un discours rituel formalisé. Dans les vallées tropicales du Vaupès colombien, les Tukano s'emparent du livre anthropologique - des recueils de mythes transcrits dans les langues tukano - et l'introduisent dans le circuit des objets sacrés pour œuvrer à la patrimonialisation de la culture. Le traitement de l'objet livre se prête alors à un déchiffrement indexical: le livre devient le signe d'une présence au même titre que les objets hérités des ancêtres (Hughh-Jones 2010 : 195-228). Perçus et maniés comme des reliques, ces textes reliés, qu'il s'agiisse de livres de mythologiie ou de bibles traduites, sont appréhendés comme des artefacts rituels. Ils matérialisent le corps des ancêtres de chaque groupe patrilinéaire exogame. Ils confèrent à leurs manipulateurs une autorité analogue à celle des aïeux, par un jeu d'emboîtement récursif. La manipulation du livre reste néanmoins indépendante de sa lecture. Elle occasionne des cérémonies où des paroles rituelles sont énoncées à voix haute par des chefs sur l'oriǵine des objets sacrés, ornements de plumes, calebasses, porte-cigares, etc. On remarque deux choses : le livre, quand il est écrit par les Blancs et qu'il enregistre la voix des ancêtres, occupe une place de choix dans le circuit des objets sacrés; et son usage semble inséparable de l'oralité.

\section{TRANSCENDANCES TEXTUELLES}

Ce n'est pas tout. Le livre peut s'affranchir de sa propre matérialité pour faire l'objet d'une appropriation virtuelle, sans que pour autant son efficacité en soit altérée. Ce n'est plus le texte lui-même, mais l'image mentale du texte et de ses avatars qui suscite la curiosité et la fascination des Amérindiens. L'écriture, comme vestige d'une technologie issue du monde blanc, devient instrument visionnaire et support de divination. En témoigne le récit de ce chamane shipibo recueilli par Angelika Gebhart-Sayer (1985: 168, traduction de l'auteure):
Durant ma vision, j'étais capable de voir la vraie nature et les vraies intentions de tous les visiteurs de ma maison: ils m'apparaissaient nus. C'est là qu'apparut l'esprit maître shahuan-peco lui-même. Il avait un livre à la main dont les feuilles étaient encore neuves et vierges. Avant de me le donner, Colibri peignit dessus de très délicats motifs à l'aide de son bec effilé. Dans un tel livre, le chamane peut apprendre des choses sur la maladie de son patient ou sur la manière de le soigner. Je lis souvent ce livre quand je chante.

La peinture des motifs délicats sur papier par l'esprit maître Colibri rappelle ce que font les chamanes shipibo pour guérir les victimes de sorcellerie: ils soufflent des chants afin de «peindre»sur leur corps des motifs kene (ibid. : 70). L'énonciation rituelle coïncide cette fois avec une lecture imaǵinaire associée à un acte de figuration. L'exemple, pris parmi des centaines d'autres, dévoile l'articulation étroite entre la perception visuelle de l'écriture alphabétique et l'appréhension des formes graphiques, de l'image et du dessin (Gow 2001: 208; Valenzuela et Valera 2005: 77; Illius 1994: 196)7. Ceci est confirmé par la présence dans plusieurs langues amérindiennes d'un champ sémantique commun, à l'instar de ces «dessins de paroles» sur «peaux de papier», «liǵnes sinueuses de l'esprit» évoquées par le chamane yanomami Davi Kopenawa dans un récit saisissant (2010 : 490-491). La lecture et l'intelliǵibilité de ces textes imaginaires sont inséparables de l'apparition d'un auteur énonciateur au rêveur. L'autonomie à l'égard de la parole virtuellement énoncée n'est donc jamais entièrement acquise. Les livres de Blancs font apparaître leurs «maîtres» par une singulière épiphanie, dont l'issue est un transfert de connaissance et d'autorité. Dans l'Oriente équatorien, le chamane zapara Bartolo Uchigua, défenseur des droits de son peuple auprès de l'Unesco, décrit la révélation onirique du «maître» d'un livre fameux, Le Contrat social :

\section{Il rêva que "l'esprit du livre » à la forme humaine - probablement l'auteur - lui recommandait de lire tout l'essai et non la partie que j'avais suggérée. Alors, quand il aurait achevé cette lecture, il pourrait discuter avec l'esprit des thèses de l'ouvrage. [...] «le rêve est comme un livre».}

(Bilhaut 2011: 223)

À quelques lieues de là, un chamane runa canelos dénommé Alfonso Chango dévoile à l'ethnologue Norman Whitten ses visions induites par la prise d'une plante hallucinogène, la stramoine arbustive wantu ${ }^{8}$, sous la forme d'inscriptions en lettres cursives gravées sur des viǵnettes photographiques: l'esprit «indique ainsi sur des tableaux, sur des photos, car pour lui, il n'existe aucun secret » (Chango et Whitten 1984: 19, fig. 3). Il s'agit cette fois de textes oniriques perçus comme des messages divinatoires. 
Ils sont écrits en espagnol et révèlent le diagnnostic de maladies, l'identité des sorciers accusés, l'annonce d'une mort imminente, etc. L'esprit maître de la petite stramoine wantu amu, auteur invisible de ces textes imaginaires, est représenté sur le dessin par un petit fantôme flottant dans l'espace de la page au-dessus de la tête du chamane, pointant de l'index les encadrés photographiques. Bien que virtuelle, la trace écrite fait ici l'objet d'un double déchiffrement. Elle se manifeste dans une perception synesthésique conjuguant stimuli visuels et auditifs et se prête simultanément à la lecture d'un texte enchâssé dans une image à la manière d'un phylactère. Si l'écriture demeure immatérielle, son apparition s'avère étroitement dépendante d'un mode perceptif spécifique: les visions ou la perception onirique.

\section{UNE ÉTRANGE BIBLIOTHÈQUE}

La bibliothèque découverte dans la maison d'EW révèle un cas d'appropriation de l'écriture alphabétique d'un genre nouveau. Quelle en est la forme exacte et comment ces textes sont-ils produits? EW écrit et archive des textes venant consigner un savoir secret et spécialisé, celui des chants rituels chamaniques ikara, prières et conjurations en langue quechua, shipibo, aents chicham ou en espaǵnol. Cette innovation suppose de la part d'EW une excellente maîtrise de la transcription alphabétique et de la lecture, acquises à l'école de la communauté où il a grandi. Cependant, à mon grand étonnement, ces écritures ne sont pas destinées à être «lues», au sens où on l'entend d'ordinaire. Il n'y a pas de corrélation entre la lecture littérale du manuscrit et la transmission du savoir dont il est question. C'est pourquoi lorsque EW m'a donné la feuille où il avait tracé mes propres paroles entendues en rêve, il m'a demandé de participer à la prochaine cérémonie d'ayahuasca qui aurait lieu le vendredi suivant et de lui souffler de la fumée de tabac sur le crâne, afin, dit-il, que le chant «pénètre joliment sa pensée» (sumakta yuyaypi yaykunanpa) et «vienne se loger à l'intérieur de son cœur» (shunkupina yaykuchinanpa). Son étrange biblioteca rassemble des papiers-paroles ou «paperoles» révélées au cours d'une vision ou d'un rêve, dont l'usage est strictement lié à ce contexte de transmission rituelle et à la possibilité d'une oralisation différée. EW inscrit au petit matin les paroles des esprits supay entendues lors de la cérémonie à laquelle il a participé, la veille. S'il ne s'aǵit pas d'une écriture automatique surréaliste, l'acte d'inscription prend néanmoins la forme d'une écriture inspirée. Il est la traduction immédiate la plus tangible d'un rêve dans lequel se dévoile l'auteur imaǵinaire de ce chant. L'écrit consigne donc une parole onirique identifiée comme celle des esprits. Les chants qui s'y trouvent inscrits seront énoncés librement plus tard, lors des cérémonies chamaniques, dans un contexte strictement rituel. La récitation donne lieu à d'importantes variations par rapport au texte écrit qui offre donc une amorce, voire un script généralement incomplet et inachevé. Une fois écrites, les paperoles ont une destinée mystérieuse. La plupart du temps archivées dans le fatras d'objets insolites que possède $\mathrm{EW}$, dans un recoin de la pièce où il officie, elles pourront être transmises à un disciple, en soufflant sur sa tête la fumée de tabac et le chant ikara qui leur échoit. La découverte dans la biblioteca d'EW de nombreux textes écrits par d'autres chamanes suggéère l'existence d'un réseau chamanique secret. À quoi ressemblent ces paperoles? Ce sont des textes rédigés en lettres cursives ou en capitales, occupant parfois tout l'espace disponible sur une page, s'il s'agit d'un carnet par exemple. Cependant, le tracé peut occasionner des remaniements du corps du texte, avec des annotations marginales, des corrections, des ratures ou des ajouts. De nombreux textes sont écrits en quechua ou dans d'autres langues, pano ou aents chicham. Sur certains rédigés en espagnol figurent des prières, oraciones, invoquant les archanges ou les saints, mêlées à des paroles de conjuration. Dans sa biblioteca, EW m'a dévoilé des écrits qui ne sont pas de lui dont la graphie est différente. Il les a obtenus auprès d'autres initiés bancu, muraya, vegetalista ou oracionista («maître des prières chrétiennes») contre paiement ou échange de techniques chamaniques et de chants. Certaines paperoles comportent des signatures étranges, parfois recouvertes de ǵribouillis, entièrement invisibilisées par l'encre ou criblées de minuscules petites flèches traversant les noms en diagonale. EW m'avoue à mi-mot que la manipulation graphique du nom est liée à un envô̂tement. Quelques pages volantes sont consacrées à l'inscription des noms de patients venus consulter pour un service chamanique (contre-sorcellerie, pacte d'amour, sortilège de fortune, etc.). À côté de certains noms, on trouve les mentions suivantes:

«Presidente», «sinchi promotor» ou «coordinador regional», indiquant la position sociale à laquelle aspire le patient.

Une fois couchés sur papier, ces chants de rêve, prières et invocations, sont manipulés avec délicatesse. EW souffle de la fumée de tabac, un parfum usé en sorcellerie nommé Tabu, acheté sur un marché local, et du liquide provenant de quelque fiole maǵique afin, dit-il, de «nourrir» (kara-) les esprits maîtres des chants et de les «faire venir» à lui quand il le désire. Un chant ikara doit «arriver seul de lui-même» (sapalla shamun), mû par son propre désir, un sentiment d'attachement envers son maître, lorsqu'il se sent «appelé » (kaya-). Si EW ne prend pas soin de ses ikara, il perd la mémoire des notes de la mélodie, du rythme des mots qui s'enchaînent dans la récitation. Son esprit s'embrouille. L'esprit maître du chant ikara ne lui apparaît plus et la petite musique talalea des esprits lui échappe. L'oubli du savoir chamanique découlerait ainsi d'une néǵligence de la relation matérielle et affective que le chamane instaure avec ces signnes. 


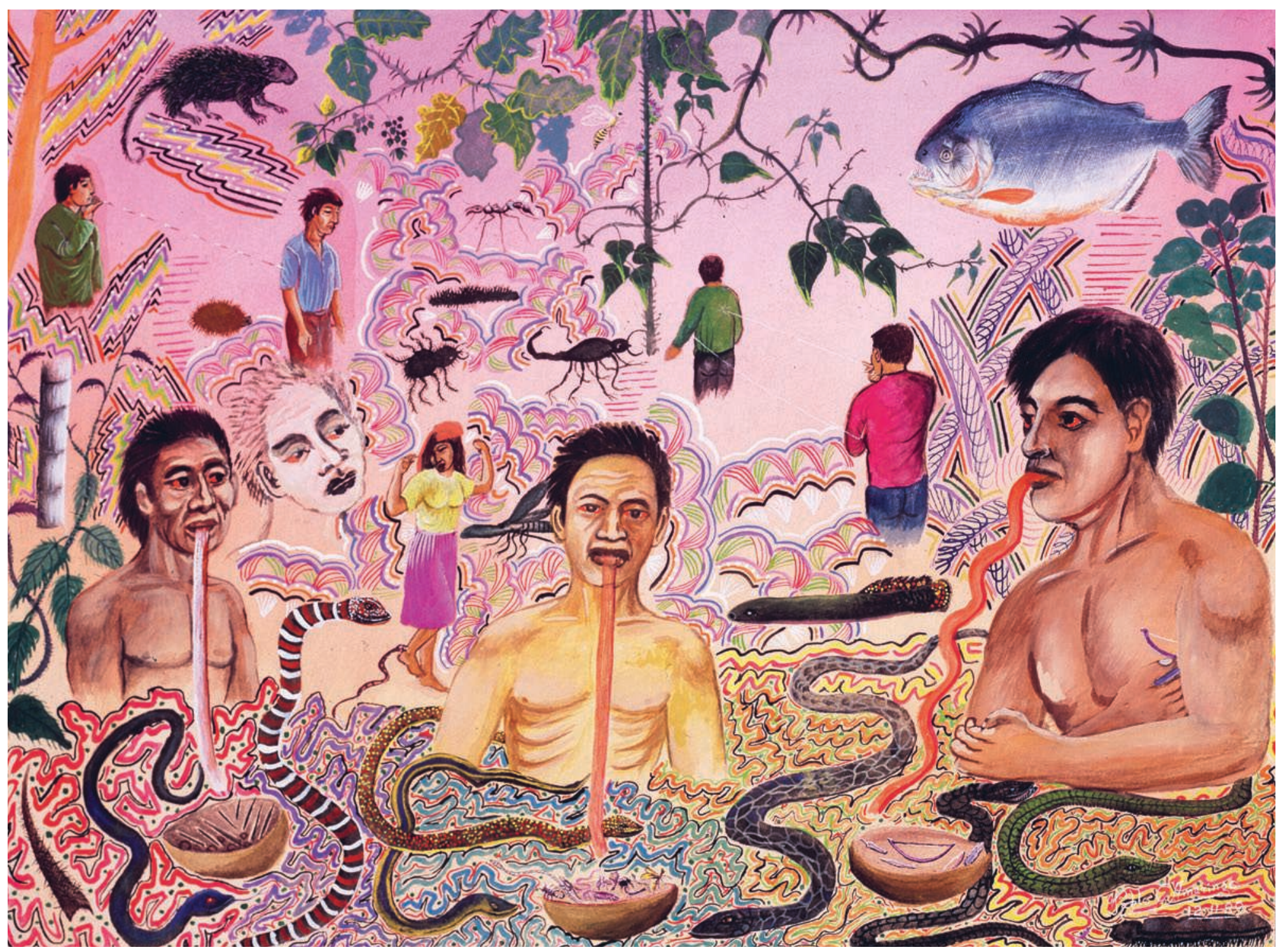

Pablo Amaringo, Trois sorciers, 1989

(c) 2021 Trueamaringos.com. Avec l'aimable autorisation de Luis Eduardo Luna. 
TRANSMISSION ET DÉRIVATION : MEMORIA VERBORUM ET MEMORIA RERUM

Qu'advient-il lorsque des peuples amérindiens, dont la mémoire semblait jusqu'alors réservée à l'oralité, ont recours à l'écriture, c'est-à-dire à la possibilité d'inscrire un savoir secret sous la forme d'une trace figée, déchiffrable indépendamment de son auteur, soit différée de son contexte d'énonciation originel? L'efficacité de cette transmission relève-t-elle d'une mnémotechnique moderne visant à stabiliser dans la mémoire du néophyte un contenu propositionnel fixe? L'écrit est-il conçu par EW comme l'objectivation matérielle immuable de la parole rituelle? Comprendre les ressorts cognitifs et pragmatiques de cette innovation, son rapport singulier à l'oralité, implique de la resituer dans le contexte du chamanisme runa. Les répertoires de chants chamaniques ikara («icaros» en espaǵnol) ou arkana relèvent d'une tradition orale qui s'étend sur une vaste région de l'Amazonie péruvienne et équatorienne, le long des axes de pénétration des fleuves Napo, Marañon, Huallaǵa, Pastaza, Corrientes et Curaray. À la différence d'autres traditions orales amazoniennes, en particulier celles du Xingu ou du Nord-Ouest amazonien, le savoir rituel des chamanes runa ne coïncide pas avec la mémorisation d'un «corpus étendu» fondé sur la répétition verbatim de certains textes, mais sur la capture continue, cumulative et incorporée de nouveaux savoirs par le rêve et les visions: il s'agit d'un «schéma génératif » de production du savoir rituel (Goody 1979; Fausto et al. 2011; Gutierrez-Choquevilea 2017). La transmission des chants rituels ikara en langue quechua se fonde sur une expérience de narcose ancrée dans la biographie des novices chamanes et induit aussi des procédés de dérivation. Des objets rituels accompaǵnent la transmission des discours, tels que des pierres nommées encantos ou aya rumi, auxiliaires «vivants» dont le chamane entend battre le «cœur», des fléchettes supay wiruti ou des substances, comme le flegme mariri, le tabac, les larves maǵiques issues des préparations végétales. L'apprentissage de la sorcellerie se matérialise par l'incorporation virtuelle de sangsues, d'insectes venimeux, de serpents ou de poissons aux dents acérées. Cette démultiplication des traces matérielles du savoir chamanique fait écho à cet autoportrait d'un chamane d'Iquitos :

Un curandero [chamane] est comme un arbre. Sous certaines conditions, il lui pousse des branches. Ces branches sont ses icaros [chants rituels].

(Luna 1986: 97)

Prolongement invisible du corps chamanique, la parole croît comme ces ramifications végétales. C'est dans la continuité de procédés figurés ou littéraux de dérivation que nous devons situer le recours à l'écriture.

\section{POLYGLOSSIE RITUELLE}

Diplomates virtuels ou effectifs, les chamanes runa yachak ou banku sillonnent la forêt pour consulter leurs homologues urishin achuar, onanya ou meraya shipibo, payum ou tsumi Cocama-Cocamilla. Un réseau interethnique secret s'est développé dans la région correspondant à l'ancienne mission de Maynas, de part et d'autre de la frontière équatorienne (Harner 1972 : 116-132; Oberem 1974 : 351; Pellizzaro 1979; Arevalo 1986; Uriarte 1989; Taylor 1981; Gnerre 2009; Muratorio 1987; Gow : 1994 ; Chaumeil 1983; Pérez 2001). Certains chamanes puissants auraient le pouvoir de disparaître en plongeant dans le fleuve Huallaǵa pour resurgír à des centaines de kilomètres de là, sur le fleuve Napo, en suivant les fils imaginaires de Nylon tendus par leurs esprits auxiliaires aquatiques. Le prestige du chamanisme runa émane du caractère attractif de la langue quechua diffusée par les missions jésuites, devenue une lingua franca connue et réputée chez de nombreux groupes ethniques de l'Oriente. Depuis plusieurs décennies, les contacts avec le monde métis et les reliǵions chrétiennes au sein des communautés ou en ville ont transformé les pratiques rituelles. En attestent l'adoption d'un nouveau genre discursif, les «prières» chrétiennes (oraciones), et d'une $p a$ raphernalia composée d'affiches ou d'objets religieux (croix, manuels de prières), de parfums achetés sur les étals de marchés locaux, de fioles de produits toxiques désinfectants (timolino, creolina) que le chamane ajoute à ses breuvaǵes pour favoriser les visions. Dans le circuit des objets rituels figurent aussi les petites pierres nommées encantos, des pipes à tabac et plus récemment des grimoires de magie (Caruso 2005: 165 ; Luna 1986: 141; Taussig 1987 : 259; Chaumeil 2003; Deshoullière 2016; Henningsen 1994; Garra 2017; Stoll 2014).

Le dynamisme de ce système chamanique macrorégional repose sur le recours à une forme de polyǵlossie rituelle. Des emprunts linguistiques sont en effet observés dans les répertoires étudiés. Il en va ainsi de mots quechua dont le sens s'est transformé par leur usage dans les chants des chamanes ennemis. Le syntaǵme tunchi désignant les «spectres des défunts» est devenu synonyme de «sorcier» chez les Aents chicham et les Awahun, tandis que le terme s'appliquant aux «esprits auxiliaires » supay dénote pour les Achuar les «chamanes ennemis». Le terme mariri, qui se réfère au fleǵme enveloppant les esprits auxiliaires à l'intérieur du corps du chamane, est devenu synonyme de «dard maléfique» pour les chamanes shipibo. La stabilisation du savoir chamanique implique donc la maîtrise de la traduction entre langues rituelles secrètes. Citons pour exemple cette paperole trouvée dans la biblioteca d'EW, sur laquelle est écrit le chant de l'Indien Chama chama, destiné à sceller un pacte d'amour. Il présente une concaténation de mots en espaǵnol, en quechua et dans une lanǵue de la famille linguuistique pano. Les syntaǵmes suivants peuvent être décryptés dans un ordre qui ne respecte la syntaxe d'aucune des langues en présence: yora («le corps»), kenay («j'appelle»), nishi («liane sarmenteuse», figuier étrangleur Ficus sp.), nuku («nous») ${ }^{9}$, juxtaposés
9. La traduction est d'EW, non locuteur de langue pano. 
à d'autres mots en quechua: warmi («femme»), kaya- («appeler»), ikara («chant»), chari («peutêtre»),jaku- («venir»).

\begin{tabular}{|c|c|}
\hline Chama chama Ikara & Ikara de l'esprit Chama chama \\
\hline Uhui uhui kaya aibu & Il appelle l'esprit uhui uhui \\
\hline Ven espiritu de warmi & Viens, esprit féminin! \\
\hline Yo kenay nukuya riki & Je t'appelle à nous \\
\hline Yo te llamo que vengas a mi & $\begin{array}{l}\text { Moi j'appelle et peut-être j'appelle } \\
\text { ton corps,viens auprès de moi! }\end{array}$ \\
\hline Yo te quiero bastante & Je t'aime beaucoup \\
\hline Tu cuerpo bonito & Ton joli corps \\
\hline Mia nishay con renaku & $\begin{array}{l}\text { Avec ma liane de ficus étrangleur } \\
\text { [Ficus sp.] }\end{array}$ \\
\hline Yo te amarro con renaco & $\begin{array}{l}\text { Je t'attache avec la liane de ficus } \\
\text { étranǵleur [Ficus } s p .]\end{array}$ \\
\hline Mia yura jaku & $\begin{array}{l}\text { Viens auprès de moi, mon joli } \\
\text { corps }\end{array}$ \\
\hline
\end{tabular}

La maîtrise de ce chant figure parmi les techniques d'envoûtement et d'attraction les plus puissantes. Son efficacité dépend de l'accomplissement d'un rituel mineur consistant à recueillir un fraǵment du corps de la personne destinataire, comme un cheveu, un onǵle coupé, ou en l'absence de tels éléments, des morceaux de tissus ayant été en contact avec la peau, pour ensuite les brûler ou les enterrer selon une pratique commune en sorcellerie. Le simple fait de permuter d'une langue à l'autre (code switching) est une technique redoutable permettant de dissimuler l'identité et l'intention du destinateur, tout en produisant un effet de stupeur chez le destinataire invisible de la parole rituelle. Qui parle? Est-ce la voix d'un chamane runa, chama ou d'un puissant curandero métis? Cette parole rituelle défiant toutes les lois de la syntaxe ordinaire se voit alors qualifiée de «parole croisée» (cruzada) particulièrement offensive, dont les chamanes ennemis peinentà déjouer les attaques. Polyǵlossie et traduction transforment le signe en une sorte de piège cognitif.

L'importance de la traduction interlinguistique dans ces paperoles fait surgiir un autre point digne d'attention: le fait que certains mots puissent être mémorisés et transmis entre chamanes sans être nécessairement intelligibles pour les locuteurs, d'une part, ou sans exister tout simplement dans aucune langue parlée, d'autre part. Il s'agit alors d'une cryptographie. C'est le cas de cette liste de noms qu'EW attribue aux dix peuples d'esprits tinkuna rencontrés en rêve par un chamane banku du Napo, qui lui en a transmis le secret:

Cakmin Shutay Ruway Shamushkay Vishta Mishquiti Shamulay Tushpa Mashqui Hitushqui Récité oralement à très vive allure par
EW, cette liste se réduit aux syllabes accentuées de chaque nom:

$$
\text { Ca...Shu...Ru...Mu...Vish...Shqui...Mu... }
$$

Tush...Mash...Tu

L'initié admet l'oriǵine inconnue de ces noms, qui cependant évoquent un air de ressemblance avec une possible étymologie quechua: $k a-$ («être»), shuti («nom»), rura- («faire»), shamu- («arriver), mishki («doux»), tushu- («danser»), mallki («arbre»). Dans la version officielle, EW s'en remet à l'expérience sensorielle qui ponctue ses propres visions pour fonder l'autorité de cette innovation par un singulier baptême visionnaire. Chaque syntaǵme isolé fonctionne comme un nom propre singularisant un être référent, un esprit rencontré dans son rêve. Ces noms viennent donc naturellement s'inscrire dans une liste ordonnée comme une suite numérale, dont la répétition doit s'effectuer selon l'ordre annoncé en rêve. L'énonciation de ces noms cryptés est perçue comme une arme supérieure à toute autre en sorcellerie. Comment interpréter la relation qui s'établit entre le chamane et le signe? Revenons à l'épistémologie de l'apprentissaǵe et au geste accompagnant l'inscription.

\section{UNE LEÇON D'ÉGRITURE GHAMANIQUE}

EW est le fils d'un père quechua originaire de la région du haut Napo et d'une mère cocama de Lagunas. Il est devenu yachak après avoir frôlé la mort suite à plusieurs échecs thérapeutiques auprès d'autres chamanes. L'enfant, accablé par des rêves terrifiants, apercevait son corps et celui de ses proches sous la forme de squelettes. Il est placé immédiatement sous la protection de son oncle, un chamane muraya puissant qui réside dans une communauté proche de la frontière brésilienne. EW raconte son initiation:

«[...] Lorsque je suis tombé malade, ma mère pleurait déjà, elle me voyait mourir. Mes oncles m'ont fait boire l'ayahuasca. Il s'en est suivi quatre années de jeûne et de réclusion. J'allais dans la forêt et j'attendais les esprits pour qu'ils viennent me guérir. Je vivais dans la tristesse et la souffrance. J'avais un mauvais sort daño fiché dans mon corps comme une flèche. J'étais incapable de respirer, je crachais du sanǵ. Je voulais mourir. J'étais en train de mourir. Je rêvais souvent et dans mes rêves apparaissaient mes grands-pères, Leoncio Waymana et Jose Curico, de vieux chamanes puissants décédés. Mon grand-père savait soigner les morsures de serpent et les mauvais sorts, dit-on. Dans un rêve, il me demande: "Tu veux apprendre?" Je réponds: "Oui". Il me demande: "Tu veux apprendre à soiǵner?" "Oui", dis-je. "Alors je vais te donner le mariri, tu vas supporter". Plusieurs personnes vinrent me voir et ils me dirent: "Ton grandpère te protège". Mais je commençais à faire des rêves de plus en plus inquiétants. J'ai pris de l'ayahuasca une nouvelle fois. Là, je rêve d'un cimetière gardé par 

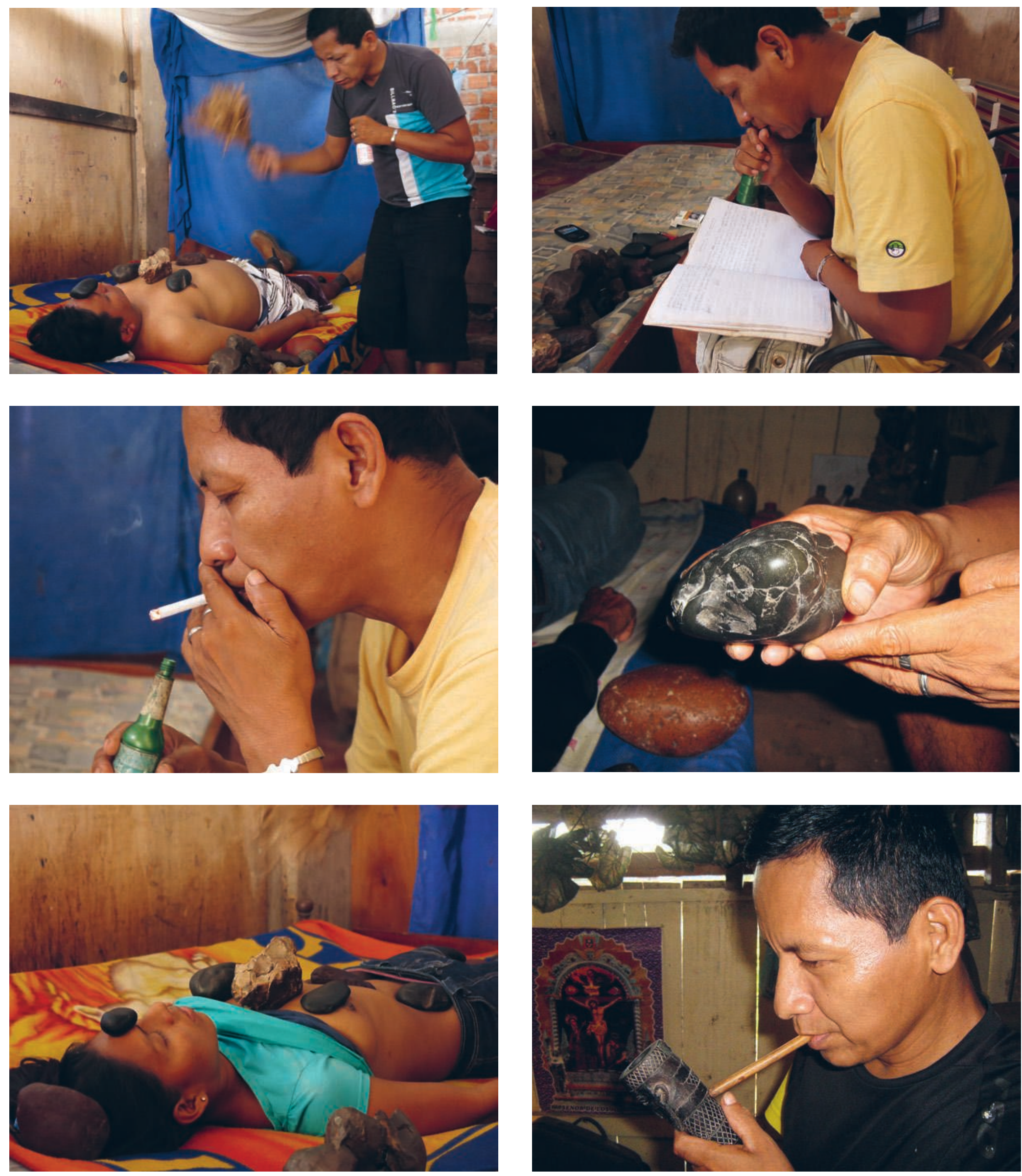

De haut en bas et de gauche à droite:

1. EW effectue un balayage «pichana» lors d'une séquence thérapeutique. Photo: Andrea-Luz Gutierrez-Choquevilca, 2011

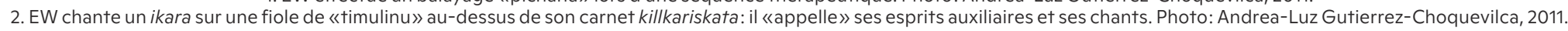
3. EW souffle de la fumée sur sa fiole de «timulinu». Photo: Andrea-Luz Gutierrez-Choquevilca, 2011.

4. Pierre auxiliaire du chamane dite de l'anaconda noir «yana yaku mama». Photo: Andrea-Luz Gutierrez-Choquevilca, 2011.

5. Séance de guérison d'une victime de sorcellerie. Photo: Andrea-Luz Gutierrez-Choquevilca, 2011.

6. EW me montre I'une de ses pipes de l'esprit Shapshiku. Photo: Andrea-Luz Gutierrez-Choquevilca, 2011 
10. Les récits visionnaires mobilisant la figure de Dieu, de la Vierge ou des saints, ainsi que des objets liés au pouvoir génératif infini du monde blanc (avions, hélicoptères, soucoupes volantes) sont légion. Luis Eduardo Luna (1986: 93) note que les chants chamaniques arkana les plus puissants sont transmis par puissants sonty transmis par Les pierres encantos employée par les chamanes d'Iquitos incarnent l'esprit de «patron», de «saint $»$ ou de «docteur». Pérez (2001: 62-63)

et Ventura i Oller (2000) signalent l'importance de prières (oraciones) insérées dans les chants rituels comme l'actualisation de «voix divines» dans le chamanisme métis ou tsachila. Un chamane runa du Napo, Quilluma, se réfère à la figure de Jesucristo pour légitimer sa position oratoire de chamane occupant le sommet de la hiérarchie chamanique régionale banku, reconnu pour son aptitude à prêter sa voix aux âmes des défunts (Muratorio 1987: 296). un prêtre avec une épée. Il me demande de traverser le cimetière. La foudre $[\mathrm{rayu}]$ m'a frappé à la tête, les esprits veulent m'enfouir dans un trou noir dans les entrailles de la terre. Là au bout, il a une maison de fer [casa de fierro] et dehors, du bétail, des vaches blanches et de couleur. Dans les arbres, un sarcoramphe pape géant me guette, menaçant. Les pièces de la maison de fer sont fermées avec des cadenas. Je suis prisonnier dans cette maison de fer. Si je reste, me dis-je, c'est que je vais mourir. Je continuais de cracher du sang.

Alors je rêve que je m'enfuis de cette maison de fer. Je voyais un petit chemin par ici qui longe un abîme où s'agitent des créatures infernales, toutes noires.

Des gens de petite taille et de grande taille accourent de partout et se rejoignent, avancent en file indienne le long de l'étroit chemin qui mène à une autre maison. Ce sont les esprits supay et les images spectrales des morts anciens aya runa. Ils me disent: "Viens par ici". J'ai répondu: "Non, je ne vais pas par là”. Je suis terrifié.

Ensuite, je devais avoir 18 ans, j'ai rêvé de Dieu et de la Vierge. J'étais en train de me diriger cette fois vers une église. Une immense maison cérémonielle faite de fils en Nylon. Elle était immense, entièrement construite par des fils lumineux tendus et croisés. Dieu a dit à d'autres personnes qui étaient à côté de moi et qui attendaient: "Ici, toi, tu vas soigner dans cette église, tu vas devenir oracionista! Tu vas jeûner pour absorber la plante sacha ahu [Mansoa alliacea]." Alors j'ai vu de mes propres yeux, à trois reprises, la Vierge. J'ai senti que je reprenais des forces.

J'ai rêvé beaucoup de fois. Les esprits supay venaient me chercher, certains d'entre eux sont terrifiants. Et là, dans mon rêve, je suis monté à un étage au-dessus, dont le sol était recouvert de nattes, de tapis de toutes les couleurs. C'est là que j'ai vu une multitude d'esprits supay qui apparaissaient avec les lettres, les lettres de Dieu. Ils surgissent de toute part puis brillent et disparaissent. Ce sont des lettres d'or. Les esprits me donnent un fil lumineux. Que fais-je avec ce fil ? Je pénètre dans la maison cérémonielle faite de fils en Nylon. Les supay essaient de m'écraser [niti-], je sens leur haleine fétide. Je pense que peut-être la fin du monde est arrivée. Des pierres enflammées tombent du ciel, de partout.

Ma bouche était froide, je ne savais ni chanter ni guérir. Alors l'esprit de Dieu m'a dit: “Tu veux te sauver? Reste! Si tu désires guérir, répète ce que je chante! Prends ces feuilles de papier et pense [yuyari-] à mes paroles." J'ai fermé les yeux et je commençai à entendre sa voix. J'étais dans l'obscurité. Alors les lettres me sont apparues comme des formes étincelantes dans l'air, elles scintillaient au-dessus de moi, ondoyant autour comme l'anaconda yacu mama. Je me suis mis à chanter ce que j'entendais. En chantant, les lettres sont apparues de nouveau cette fois, elles se transforment et prennent la forme des esprits supay. Leurs corps brillent. Elles forment des traits et des ziǵzaǵs, des ondulations, ce sont les tinkuna [lettres de «protection» maǵiques]. Elles peuvent tuer leurs ennemis sorciers avec ça. Les lettres s'élèvent et ondulent. Saaa kinku kinku saaa saaaa [son du mouvement des lettres qui s'élèvent dans l'air]. Elles me fixent du regard. Je sens le parfum de leur corps; elles se dressent devant moi, sous mes yeux, très clairement! Je me suis mis à écrire. Le lendemain et les jours suivants. [...] Tout cela est arrivé après avoir traversé cette grande affliction. C'est ainsi, après avoir jeûné, après avoir souffert, puis absorbé les plantes de la forêt dans mon corps, je rêve... Ces créatures me font rêver et le lendemain, j'écris ce que j'ai entendu. C'est ainsi que l'on apprend.»

Ce qui s'apparente à une catabase infernale dans un espace associé au monde des âmes spectrales aya runa correspond à la variante d'un parcours initiatique commun. L'apprentissage est ponctué d'épisodes de souffrance causés par des visions terrifiantes, la maladie (unkuy), le jeûne (sasi-), voire par une quasi-mort (wañu-). La «maison de fer» (casa de fierro), prison imaginaire d'EW, est une allusion à l'édifice bâti à Iquitos d'après les plans architecturaux de Gustave Eiffel, à l'initiative d'un baron du caoutchouc espaǵnol, Anselmo del Aǵuila, venu visiter l'Exposition universelle de Paris en 1889. Elle situe donc la révélation des "créatures lettres» divines tinkuna aux temps de l'exploitation de la main-d'œuvre indigè̀ne par les patrons blancs, dans un espace semi-urbain composé d'étages successifs entièrement cousus par des fils en Nylon lumineux. Ceux-ci évoquent les fils de pêche sur lesquels les âmes des chamanes sumi runa et muraya se déplacent sous l'Amazone. Le point culminant du récit est une scène apocalyptique dans laquelle apparaissent la Vierǵe puis Dieu en personne, sommant l'initié de répéter ses paroles, de «penser» et de les écrire. La figure de «Dieu» vient occuper une position d'initiateur analogue à celle d'autres esprits émanant du monde blanc, tels ces docteurs (mediquitos) et infirmières blonds débarqués d'un bateau à vapeur fantôme aceropunta ou d'une soucoupe volante, qui hantent les visions des chamanes runa, métis ou yine (Luna et Amaringo 1999: 40 et 86; Gow 2001) ${ }^{10}$. L'avènement mythique des écritures ikara ouvre une passerelle temporelle vers la période traumatique du règne des barons du caoutchouc sur l'Oriente, époque d'esclavage et de désolation, dont les spectres aya runa sont aujourd'hui les seuls "témoins». L'initiation s'apparente donc à une étrange rétrospective sur l'événement de la rencontre et de l'intensification des contacts - meurtriers - avec les Blanes. La leçon d'écriture révélée à $\mathrm{EW}$ contient les germes utopiques d'une subversion de la violence colonisatrice.

\section{LA LECTURE IMAGINAIRE}

Comment les chants ikara sont-ils mémorisés? La stabilisation de la parole rituelle dans la mémoire de l'initié emprunte plusieurs voies: la répétition d'une parole onirique, la lecture d'un texte et la contemplation, soit un acte de subjectivation mutuelle 
de l'initié et des esprits. La singularité de ce dispositif tient à la relation au savoir initialement conçue par EW comme une lecture imaginaire, dont les conditions sont fixées par ses visions. Il lui faut lire et entendre des voix pour devenir à son tour écrivain. Le déchiffrement de lettres alphabétiques coïncide avec l'apparition des esprits supay révélés sous la forme de noms tracés et de corps. Cette lecture s'apparente à un acte perceptif synesthésique caractérisé par des sensations auditive, visuelle, olfactive. À mesure que se déroule sous les yeux d'EW la transformation des «lettres étincelantes ondoyant comme l'anaconda», formant des traits (killka-), des ziǵzaǵs (kinku) et des ondulations (muyuri), avant de revêtir un corps et une apparence humanoïde, il perçoit leur voix comme d'intenses sifflements. Sur le plan iconographique, ces textes imaǵinaires composés de traits et de tortillons colorés évoquent les motifs abstraits tinkuna des peintures de visions chamaniques élaborées par le chamane Pablo Amaringo (Luna et Amaringo 1999: 133-135). La variation continue de ces doubles imaǵinaires de l'écrit retient l'attention. Les entités révélées, tinkuna, supay, aya runa, sont en effet campées dans une position indéterminée, entre la catégorie de sujet et celle de signe, saisi et interprété dans un face-à-face entre l'initié et son maître divin. Le geste d'inscription qui a lieu au réveil se confond quant à lui avec un dévoilement du corps des esprits, désiǵnés par le tracé de leur nom. «Voici l'ikara du vanneau de cayenne timelito, et là, le spectre Shapshiku, voici l'Aïeul, et ici le ficus étrangleur... voici ton chant, Andrea-luz », murmure EW en me montrant ses paperoles. L'écrit est alors l'élément tangible de cette révélation d'une voix inarticulée à l'oriǵine d'un chant, comme en atteste la transcription de nombreuses icônes sonores dans les paperoles: «uhuy» «llipia llipia», «den den den den», «day day day day» «kun kun kun». Ce sont les citations des voix non humaines entendues en rêve. La polyphonie du texte rend par conséquent lisible la métamorphose du signe, dans un rêve où la petite musique des esprits se déploie spontanément en paroles articulées ou en lettres lumineuses. Lors de la cérémonie d'ayahuasca, les esprits des substances végétales ingérées par l'initié effectueront leur danse de présentation rythmée par ces icônes sonores, comme en témoigne cet ikara énoncé puis retranscrit par EW :

$\begin{array}{ll}\text { suma suma runasitu } & \text { Petit esprit à la beauté radieuse } \\ \text { Aya runa runasitu } & \begin{array}{l}\text { Petit esprit image spectrale } \\ \text { du défunt }\end{array} \\ \begin{array}{l}\text { shamurimun tushurimun } \\ \text { Elle arrive elle danse } \\ \text { "day day day day day day» }\end{array} & \begin{array}{l}\text { Petite sœur de l'Aïeul [antiguo] } \\ \text { ONOM [danse de présentation }\end{array} \\ & \text { des esprits] } \\ \text { aya runa runasitu } & \text { Petit esprit image spectrale } \\ \text { shamurimun «kun kun kun } & \text { du défunt, } \\ \text { kun» shayarin } & \text { Il arrive ONOM [pas] il se dresse } \\ \text { ayawasca sankusitu awanshi } & \text { Sur l'épais breuvage de l'ayahuasea, } \\ \text { "ging ging ging ging» } & \text { dit-on, ONOM } \\ & \text { [danse sur le breuvage maǵique] }\end{array}$
luntachirimunchi «deng deng»
shapshikitu runasitu
suma suma runasitu
Nous les faisons plonger dans les profondeurs ONOM
Petit esprit de la forêt Shapshiku Petit esprit à la beauté radieuse

shamurimun tushurimun

paninchi del antigui

shapshikitu runasitu

shamurimun sankusitu

aroanshi

«chin ǵing ging ǵing»

muntachirimun

"chin ging ging ging day

day day'da naynay nay riii»

piña piña runasitu

Elle arrive elle danse

Notre sœur de l'Aïeul

Petit esprit de la forêt Shapshiku,

Il arrive au-dessus de l'épais

breuvage dit-on

ONOM il s'amoncelle

ONOM [danse sur le breuvage maǵique]

$\begin{array}{ll}\begin{array}{ll}\text { Chakinampanaya trankilimun } \\ \text { chakinampanaya piya piya }\end{array} & \text { Afin qu'il désire désire sécher, il s'apaise } \\ \text { runasitu } & \text { esprit de l'oiseau pia pia } \\ \text { shamurimun tushurimun } & \text { Elle arrive, elle danse } \\ \text { warmisita del antigui } & \text { La petite femme de l'Aïeul } \\ \text { dari ri ri ri ri ri ri ri ri } & \text { ONOM [danse et chant de l'oiseau] }\end{array}$

munanapayana warmisita yura yura renakitu

yura yura renakituwanshi

Son désir acéré, la petite femme Blanche, la liane de Ficus blanche Blanche, avec la liane de Ficus

blanche, dit-on,

armachirimunchi

nous l'immergeons dans l'eau

"naynay naynay naynay dii

dii... day day day»

chirimunchi

yura yura renakita

warmisita

«day day day day»

«daydaydayda»

shapshikitu

runasitu suma suma

runasitu shamurimun

tushurimun panisitu

"naynay naynay naynay

dii dii»

maririri maririri

shamurimun tushurimun

panisita del antiguo

shapshikitu runasitu

shayarimun shamurimun

sankunsitu arwanshi

ONOM [chant des esprits]

Refroidissons-le

Blanche, petite femme de la liane

de Ficus toute blanche

ONOM [danse des esprits]

ONOM [danse des esprits] Petit spectre de la forêt Shapshiku

Petit esprit à la beauté irradiante

Petit esprit qui arrive

Petite sœur qui danse

ONOM [chant des esprits]

Fleǵme mariri mariri

Elle arrive, elle danse vers moi

La petite sœur de l'Aïeul

Petit spectre de la forêt Shapshiku

Il se dresse, il arrive sur l'épais

breuvage, effleurant sa surface,

dit-on

La manipulation des paperoles révèle d'autres aspects importants, notamment les qualités iconique et indexicale de ces signes. Corrections et ratures sont explicitement conçues comme les empreintes ou «signatures» (firmas) des esprits révélés. Elles combinent de fait ces quatre éléments : la fonction individualisante d'un nom propre, l'effet de présence d'un graphisme tracé à la main, la saillance visuelle d'un signe personnel et la force d'un acte de langage. Les corrections attesteront de l'authenticité du savoir 


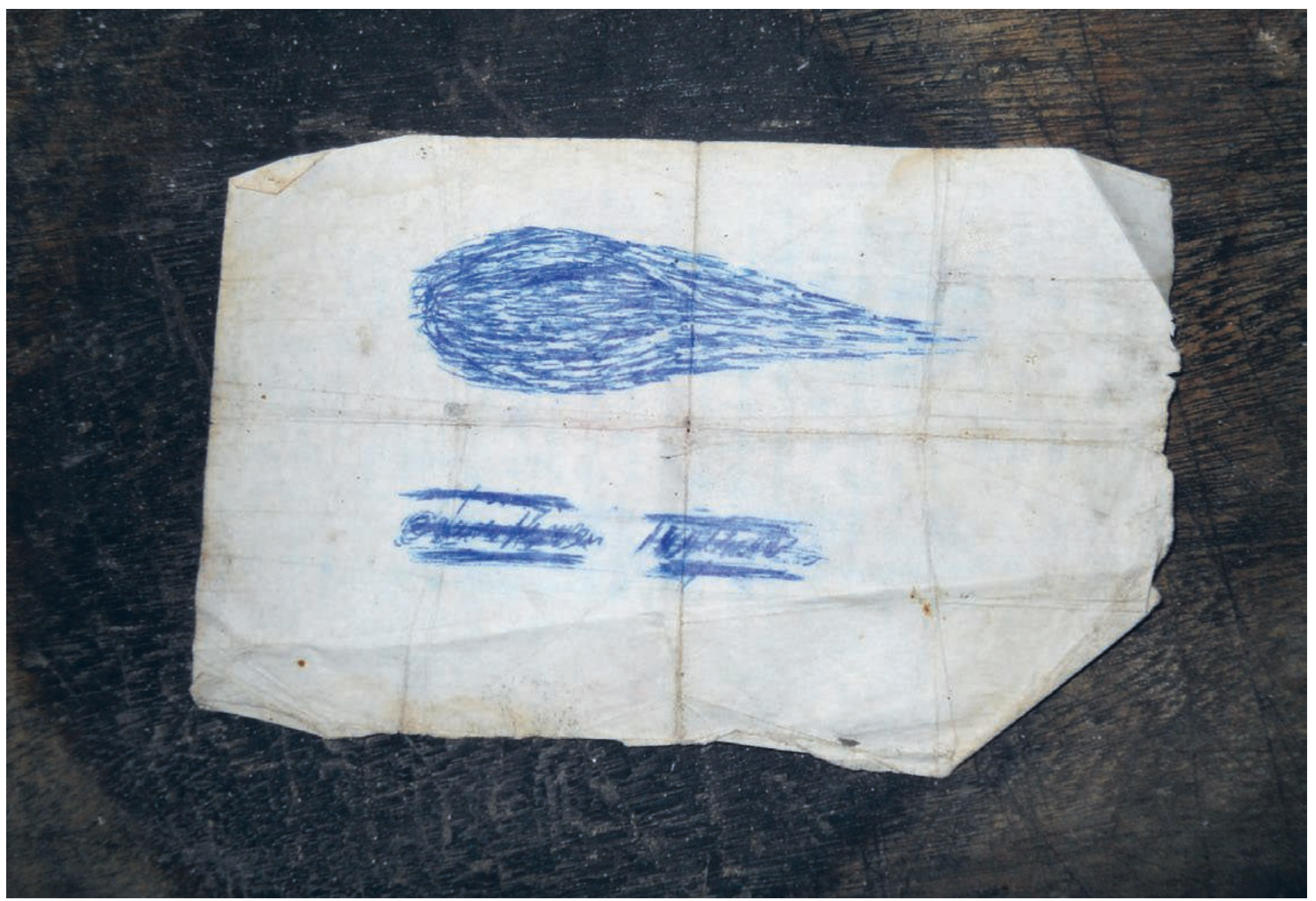

Forme iconique d'un chant ikara révélé dite firma («signature»), et noms de patients barrés. Photo: Andrea-Luz Gutierrez-Choquevilca, 2011.

11. Pour un traitement similaire des noms propres dans les livres de magie en Équateur, voir: Wogan 1998:189. entendu. L'écrit se voit ainsi doté des propriétés sémiotiques d'une signature encodant une relation à la validité et à l'identité du savoir (Fraenkel 1992: 174). Les paperoles d'EW portent la trace d'un événement, d'une rencontre avec les esprits maîtres et leur inscription vient sceller une relation charnelle entre le disciple et son maître. Celle-ci se prolonge après l'acte ponctuel d'inscription. Une sorte de «métabolisme» rituel est en effet attribué au texte: les signnes écrits sont «nourris» (kara-) avec de la fumée de tabac, du parfum et des paroles chantées ou sifflées. EW insuffle ses chants qu'il murmure à voix basse sur la surface plane du papier et aǵite son hochet chamanique shinku shinku sur le texte lors de la récitation. Il s'enduit les mains de plantes odorantes mishki alwaka, avant de manipuler les feuilles des manuscrits. Par contraste avec le soin réservé à la conservation et à l'archivage de noms d'esprits révélés, l'inscription des noms des patients ne peut se faire qu'une seule fois, au pied ou en tête des manuscrits, et doit être à terme invisibilisée. Elle est la trace éphémère d'un pacte scellé avec celui qui porte ce nom. Une fois la guérison ou le charme obtenu, ces noms seront barrés et soignneusement effacés au point de devenir illisibles ${ }^{11}$. Le traitement spécial de ces écrits laisse supposer qu'ils sont envisagés, dans la continuité du traitement accordé à d'autres artefacts rituels tels que les pierres chamaniques incarnant les auxiliaires du chamane, comme dotés d'une «vie». Le support mnémonique, objectivation concrète de la parole vive - l'inscription figée sur papier - donne ainsi lieu à un processus de subjectivation de l'écrit-texte: les esprits supay sont les «lettres de Dieu», des corps tracés des esprits, entre l'imaǵe figurative, l'index et le signe linguistique.

\section{LE CHAMANE ÉGRIVAIN}

Quel est, dans ce contexte précis, le statut du chamane écrivain? La position du yachak se voit tout d'abord suspendue entre celle d'un énonciateur de la parole vive et celle d'un scribe virtuellement détaché de sa production. Le chamane yachak est à la fois auteur et simple porte-parole des esprits, exécutant de la transmission. Il n'accède au statut de maître coïncidant avec celui de lecteur et d'éventuel auteur, suceptible d'apposer sa propre signature sur une paperole, qu'après avoir observé le jeûne, l'abstinence, l'ingestion de breuvages psychotropes et la quête de visions, soit une ascèse longue et douloureuse.

Les circonstances de la transmission confirment ce lien indissoluble entre l'action rituelle et le déchiffrement sémiotique complexe du texte. Les paperoles passent d'un chamane à l'autre lors d'un bref rituel au cours duquel le précepteur souffle du tabac sur le crâne de l'initié en murmurant les paroles chantées au-dessus de sa tête. Il souffle ensuite de la fumée sur ses yeux clos qu'il dit «ouvrir» au regard des esprits supay. 
La transmission du support écrit semble ainsi étroitement liée à une forme de transmission typiquement orale.

On peut s'interroger sur les raisons de la stabilisation de cette technique et sur la nécessité d'avoir recours à un tel régime spécial de l'écrit. Quel intérêt mnémonique y a-t-il à coucher sur papier un savoir hautement spécialisé dont l'oralité est parvenue jusqu'à présent à garantir la persistance dans le temps? La traduction des ikara en paperoles n'a-t-elle pas pour corrélat une trahison des principes essentiels de la mémoire orale? On pense notamment au principe de variation de la parole vive et à la relation de coprésence qui caractérise le cadre de l'énonciation. Un premier point à noter est le suivant. Les paperoles chamaniques ne semblent pas avoir eu pour objectif la stabilisation pérenne d'un savoir figé sous une forme immuable et détachée de son auteur ou de son contexte d'inscription. Au contraire, la lecture de ces textes est venue s'enchâsser dans un dispositif de transmission dont la particularité est de se fonder sur le principe de la permutation sémiotique: la possibilité simultanée de «lire» un même signe sous des modalités variables, de percevoir la transformation subtile d'une image figurative en signe visuel tracé, puis en texte, par un jeu de cryptage et de décryptage élémentaire du savoir. Il est nécessaire d'insister sur la sémiotique hybride qui caractérise la production de ces textes. À la manière d'un palimpseste, chaque chant ikara dévoile des niveaux de savoir différents, indexant toutes les relations possibles que le sujet est susceptible d'entretenir avec le signe. Le va-et-vient continu entre un hypotexte onirique, une parole énoncée et chantée dans un rêve, un hypertexte et une parole oralisée avec des variations démontre cette instabilité sémiotique originelle qui caractérise l'appropriation chamanique de l'écriture alphabétique. Le geste d'inscription et le savoir inscrit perdraient toute valeur s'ils n'étaient accompagnés d'une expérience sensorielle et poétique. La permutation entre l'icône, l'index, le symbole, c'est-à-dire entre le chant, la parole articulée et l'imaǵe, est la condition qui assure l'efficacité du discours rituel. L'écriture serait ainsi la manifestation tangible d'une voix révélée, sans qu'aucun canal sémiotique exclusif ne parvienne à épuiser la signification du savoir qu'elle transmet.

Un second point concerne le type d'autorité dont cette innovation a pu se prévaloir, pour s'intégrer dans le savoir traditionnel des chamanes amazoniens. On sait que la plupart des répertoires chamaniques sont appris sur le mode de la citation, du discours rapporté de la voix des esprits. L'efficacité de la parole rituelle dérive de l'attribution d'une autorité au chamane et aux énonciateurs oriǵinels de cette parole, à travers une identification de leurs voix ${ }^{12}$. Lidée que le recours à l'écrit pourrait court-circuiter ce dispositif, au profit d'une autonomie accrue du «texte-parole» détaché de son/ses auteur/s et de son contexte de transmission, est improbable. En effet, l'efficacité rituelle prêtée au signe chamanique repose ici précisément sur le lien personnel et substantiel qui se tisse entre un auteur, un énonciateur imaginaire et actuel. Il me fallait immanquablement souffler sur la tête de mon ami de la fumée de tabac lors d'une cérémonie pour que mon chant lui soit définitivement transmis : «ouvrir» ses yeux et son crâne pour y introduire ma pensée et mon chant. L'objet de la transmission est donc moins la capacité à «déchiffrer» un texte en écriture alphabétique que cette autorité acquise au cours de l'initiation, d'une part, et ce rapport complexe au signe linguistique, d'autre part. On entend par là une relation au savoir et au processus de transmission, inséparable de l'ancrage corporel, gestuel et de la coprésence qui fait de l'initié l'unique herméneute du savoir chamanique ${ }^{13}$. L'adoption de l'écriture dans ce contexte ne paraît fonder sa légitimité pour s'intégrer dans le champ du rituel que par ce lien indissoluble qu'elle tisse avec le canal de l'oralité.

$*$

Qu'est-ce qu'un siǵne qui présente de multiples lectures possibles et dont l'efficacité suppose une relation imaǵinaire avec un double du signne rêvé ou fantasmé? La double enquête menée dans ce texte par un chamane runa et une anthropologue dévoile les variations du déchiffrement et de l'appropriation de l'écriture par les Amérindiens. S'il est prêté aux chants chamaniques ikara le pouvoir de sceller des pactes, de guérir ou de transformer l'ordre du monde, le signe écrit est devenu dans le chamanisme d'EW la trace d'un corps, celui des petites âmes aya runa et des esprits supay. Une conception radicale de la traduction, opérant tant sur des signes que sur des êtres, révèle une ontologie du langage fondée sur la transformation. En faisant de l'ethnologue la compaǵne d'un rêve et une source d'inspiration poétique, EW a trouvé un moyen de supplanter les frontières cognitives et symboliques qui nous séparent. L'écriture ouvre la voie d'une utopie sémiotique. Tout en indexant un mode de connaissance explicitement dérivé du pouvoir des Blancs, la technologie des paperoles mobilise une herméneutique d'une complexité égale à celle qui préside à la lecture de l'image rituelle. Elle ne peut être saisie sans recourir à une opération de traduction. L'écriture comme réflexivité du signe, distance à soi et re-présentation est venue se greffer sur un système de dérivation préexistant dans l'épistémologie du savoir rituel amérindien, mobilisant dans une série de variations infinies la parole, l'objet et l'image. En raison de sa diffusion restreinte et de sa spécificité praǵmatique, cette innovation amérindienne n'est donc pas venue se substituer aux formes de mémorisation orales d'Amazonie. L'hypothèse d'une «domestication» de la pensée sauvage se voit ici réfutée empiriquement. L'éniǵme qui se pose est celle d'une écriture sauvage, d'une utopie sémiotique fondée sur l'instabilité et la permutation.
12. Devenir chamane, c'est en un sens précis «devenir esprit», voir: Graham 1994 Déléage 2009. GutierrezChoquevilca 2011; Cesarino 2016.

13. Une analyse comparable du rapport entre médiation chamanique et technologie numérique est faite par Pedro Cesarino chez les Marubo (2019:180). 


\section{Bibliographie}

Azevedo de Abreu, Stela

2004 Aleluia e o banco de luz: messianismo indíona no norte amazônico. Campinas, Centro de Memória-Unicamp.

\section{Allard, Olivier}

et Walker, Harry (dir.)

2016 Dossier «Amazonian Appropriations of Bureaucracy and Documents », Journal of

Latin American and Carribean Anthropology 21 (3) : 402-496.

\section{Arevalo, Valera Guillermo}

1986 «El ayahuasca y el curandero Shipibo-Conibo del Ucayali (Perú)», América Indígena 46 (1) : 147-162.

\section{Axtell, James}

1985 The Invasion within: The Contest of Cultures in Colonial North America. Oxford, Oxford University Press.

\section{Bacigalupo, Ana Mariella}

2014 "The potency of indigenous "Bibles" and Biographies. Mapuche Shamanic Literacy and Historical Consciousness American Ethnologist 41 (4) 648-663.

\section{Bilhaut, Anne-Gaël}

$2011 \mathrm{El}$ sueño de los sáparas: patrimonio onírico de un pueblo de la alta Amazonía. Quito, Abya Yala /Flacso.

\section{Brown, Michael F.}

1993 «Facing the State, Facing the World: Amazonia's Native Leaders and the New Politics of Identity », L'Homme 33 (126-

128): $307-326$

\section{Butt-Colson, Audrey}

1971 «Hallelujah among the Patamona Indians », Antropologica 28 : 25-58.

\section{Caruso, Giuseppe}

2005 Onaya Shipbo-Conibo: sistema médico tradicional y desafíos de la modernidad. Quito, Abya-Yala

\section{Cesarino, Pedro N.}

2016 «Cartographies du cosmos. Image, parole et savoir chez les Marubo », in Carlos Fausto et Carlo Severi (dir.), Paroles en images, écritures, corps, mémoire. Marseille, OpenEdition Press 2019 «Diverǵence technologique. Chamanisme et numérisation en Amazonie, Gradhiva 29 (1) : 162-187.

\section{Chaumeil, Jean-Pierre}

1983 Voir, savoir, pouvoir le chamanisme chez les Yagua du Nord-Est péruvien. Paris, Éditions de l'EHESS.

2003 [1992] «Chamanismes à géométrie variable en Amazonie», Diogène : 159-175.

\section{Chango, Alfonso} et Whitten, Norman E.

1984 Yachaj sami yachachina, Carmen Chuquín et Sibby Whitten (dir.). Quito, Abya Yala.

\section{Colpron, Anne-Marie}

2004 «Dichotomies sexuelles dans l'étude du chamanisme: le contre-exemple des femmes chamanes shipibo-conibo». Thèse de doctorat en ethnologie, université de Montréal.

\section{Déléage, Pierre}

2009 Le Chant de l'anaconda. l'apprentissage du chamanisme chez les Sharanahua (Amazonie occidentale). Nanterre, Société d'ethnologie. 2010 "Rituels du livre en Amazonie», Cahiers des Amériques latines 63-64: 229-250.

2017 Lettres mortes: essai d'anthropologie inversée. Paris, Fayard.

\section{Deshoullière, Grégory}

2016 «Vanités aux livres. Variations magiques en pays jivaro », Hypalampuses hemeras - À la pointe du jour (Revue Gruppen): 42-55.

Fausto, Carlos et al.

2011 «Les formes de la mémoire: art verbal et musique chez les Kuikuru du haut Xinǵu (Brésil)», L’Homme (197): 41-70.

Favret Saada, Jeanne

1990 «Être affecté», Gradhiva, $8: 3-9$

\section{Fraenkel, Béatrice}

2012 La Signature: genèse d'un signe. Paris, Gallimard.

\section{Garra, Simone}

2017 «Les sorciers condamnés chamanisme et mutation dan le monde awajún (Amazonas, Pérou).» Thèse de doctorat en ethnologie, université Paris X.

\section{Gebhart-Sayer, Angelika}

1985 «The Geometric Designs of the Shipibo-Conibo in Ritual Context », Journal of Latin American Lore 11 (2) : 143-175.

\section{Gnerre, Maurizio}

2009 «While I Sing, I Am Sitting in a Real Airplane: Innovative Contents in Shuar and Achuar Ritual Communication ", in Ellen Basso et Günter Senft (dir.),

Ritual Communication. Oxford/ New York, Berg Publishers: 293-316.

\section{Goody, Jack}

1977 «Mémoire et apprentissage dans les sociétés avec et sans écriture. La transmission du Bagre», L’Homme 17 (1): 29-52. 1979 La Raison graphique: la domestication de la pensée sauvage. Paris, Le Sens commun.

\section{Gordillo, Gaston}

2006 "The Crucible of Citizenship: ID-Paper Fetishism in the Argentinean Chaco ", American Ethnologist 33 (2) 162-176.

\section{Goulard, Jean-Pierre}

2000 «La parole et le livre dans la configuration religieuse d'Amazonie. Chamanisme et messianisme chez les Tikuna», in Denise Aiǵle et al. (dir.), La Politique des esprits: chamanismes et religion universalistes. Nanterre Société d'ethnologie: 267-294.

\section{Gow, Peter}

1994 «River People: Shamanism and History in Western Amazonia», in Nicholas Thomas et Caroline Humphrey (dir.), Shamamism, History and the State Ann Harbor MI, The University of Michigan Press : 90-113.

2001 An Amazonian Myth and Its History. Oxford, Oxford University Press.

2009 «Christians. A Transforming Concept in the Peruvian Amazon", Robin Wright et Aparecida Vilaca (dir.), Native Christians: Modes and Effects of Christianity among Indigenous People of the Americas. University of Florida Press: 33-52.

\section{Graham, Laura}

1994 «Dialoǵic Dreams: Creative Selves Coming into Life in the Flow of Time», American Ethnologist 21 (4): 723-745.

\section{Gutierrez-Choquevilca}

\section{Andrea-Luz}

2011 «Sisywawaytii tarawaytii. Sifflements serpentins et autres voix d'esprits dans le chama-

nisme quechua (Pastaza)»,

nisme quechua (Pastaza) américanistes 97 (1) : 179-221. 2017 «La contaǵion des imaǵes voix enchâssées et dispositifs sensoriels dans un rituel chamanique amérindien », Hybrid. Revue des arts et médiations humaines 4 [en ligne], disponible sur: https://hybrid.univ-paris8. fr/lodel/index.php?id=759 (consulté le 11 février 2021).

\section{Guzmán-Gallegos, \\ María Antonieta}

2009 «Identity Cards, Abducted Footprints, and the Book of San Gonzalo. The Power of Textual Objects in Runa Worldview», in Fernando Santos-Granero (dir.), The Occult Life of Things Native Amazonian Theories of Materiality and Personhood. Tucson, The University of Arizona Press.

\section{Harner, Michael J.}

1972 The Jivaro: People of the Sacred Waterfalls. NewYork, Anchors Books

\section{Henningsen, Gustav}

1994 «La evanǵelización neǵra difusión de la magia europe por la América colonial», Revista de la Inquisición 3: 9-27.

Hugh-Jones, Stephen 1992 «Yesterday's Luxuries, Tomorrow's Necessities: Business and Barter in Northwest Amazonia», in Caroline Humphrey et Stephen

Hugh-Jones (dir.), Barter, Exchange and Value: An Anthropological Perspective. Cambridge, Cambridge University Press : 42-74.

2010 «Entre l'image et l'écrit: la politique tukano de patrimonialisation en Amazonie», Cahiers des Amériques latine, 63-64: 195-228.

\section{Illius, Bruno}

1994 «La Gran Boa: Arte y Cosmologia de los Shipibo-Conibo », Amazonia Peruana 24 (12) : $185-212$

\section{Kopenawa, Davi}

et Bruce, Albert

2010 La Chute du ciel: paroles d'un chaman yanomami.

Plon, Paris

\section{Leavitt, John}

2005 «L'analyse des rêves », Gradhiva 2 109-124.

\section{Lévi-Strauss, Claude}

1955 Tristes Tropiques. Paris, Plon.

\section{Luna, Luis Eduardo}

1986 Vegetalismo: Shamanism among the Mestizo Population of the Peruvian Amazon. Stockholm Almqvist \& Wiksell Internationa

\section{Luna, Luis Eduardo}

et Amaringo, Pablo

1999 Ayahuasca Visions: The Religious Iconography of a Peruvian Shaman. Berkeley 


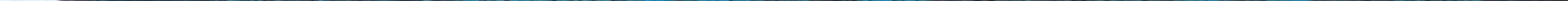

